\title{
L'uso appropriato di antibiotici iniettivi a domicilio: criteri epidemiologici, clinici e farmacoeconomici
}

RICERCA ORIGINALE

Mario Eandi*, Roberto Serra ${ }^{\circ}$

\begin{abstract}
Outpatient parenteral antibiotic therapy (OPAT) has become an increasingly used therapeutic modality during the last 20 years. Reasons for this rapid growth include OPAT's many patient benefits as well as the development of new technologies and well-documented cost savings.

Recently the Italian Drug Agency (AIFA) has revised the list of drug's limitations and indications; particularly two notes about OPAT (n. 55- n.56) have been modified. These new notes can assume at least a double significance: if on the one hand they try to put a check on the overuse and misuse of OPAT, steering physicians towards a better utilization of parenteral antibiotics, on the other they allow to prescribe OPAT also in case of non-ordinary infections, promoting development of more structured OPAT programmes.

Prescribing physicians should be aware of a number of aspects of OPAT which distinguish it from other forms of therapy: multiple factors must be taken into account, including the probable infecting organism, the pharmacodynamic and pharmacokinetic properties of candidate drugs, the capabilities of patients who will receive OPAT and of their caregivers.

The Infectious Diseases Society of America (IDSA) Guidelines have been elaborated to help physicians thorough assessment of OPAT suitability, even if they need to be adapted to many variables in each treatment setting.

Aim of the present study is to re-consider the main microbiologic, clinical and pharmacoeconomic criteria supporting the prescription of an outpatient parenteral antibiotic therapy after the publication of the new AIFA notes.
\end{abstract}

Keywords: outpatient parenteral antibiotic therapy, OPAT, microbiologic, clinical and pharmacoeconomic criteria Farmeconomia e percorsi terapeutici 2005; 6 (1): 37-56

\section{INTRODUZIONE}

L'utilizzo da parte del medico di Medicina Generale degli antibiotici iniettabili in ambito extra-ospedaliero viene fortemente disincentivato, in Italia, da due differenti note AIFA (ex note CUF), che vincolano la prescrizione a carico del SSN rispettivamente a specifiche condizioni cliniche (nota 55) o alla necessità di garantire la continuità terapeutica ospedale-domicilio (nota 56), nel caso di terapie iniziate in ospedale (tabella I).

Le giustificazioni addotte per introdurre queste note e le relative limitazioni sono di varia natura e comprendono la necessità di razionalizzare l'uso degli antibiotici sul territorio e di contenere la spesa farmaceutica pubblica, la preoccupazione di limitare il rischio d'insorgenza e diffusione di nuove resistenze agli antibiotici, l'opportunità di riservare ai medici ospedalieri l'utilizzo di alcuni potenti e poco maneggevoli antibiotici parenterali per salvaguardare il più a lungo possibile la loro elevata efficacia terapeutica e per ridurre il rischio di effetti tossici correlabili alla loro criticità d'uso [1].

Secondo stime dell' Osservatorio Nazionale sull'Impiego dei Medicinali (OsMED), la prescrizione degli antibiotici iniettabili soggetti a nota CUF, globalmente considerati, ha continuato a manifestare un sensibile trend negativo nel corso del 2003, con un consensuale risparmio sulla spesa farmaceutica. Tale risultato è attribuibile principalmente al netto calo nella prescrizione extra-ospedaliera di aminoglicosidi (-26,8\%) e ad un sensibile calo nella prescrizione delle penicilline $(-12 \%)$. Tuttavia, nello stesso periodo, è diminuita la prescrizione di cefalosporine di $\mathrm{II}^{\circ}$ generazione ed è aumentata quella di cefalosporine di $\mathrm{III}^{\circ}$ generazione e, nell' ambito delle penicilline protette, è calata la prescrizione di ticarcillina associata ad inibitori enzimatici, mentre è aumentata la prescrizione di piperacillina/tazobactam [2].
* Cattedra di Farmacologia Clinica, Università degli Studi di Torino

- Laboratorio

Microbiologia Clinica, Azienda Ospedaliera S. Giovanni Battista, Torino 
Nei primi sei mesi del 2004 i consumi di antimicrobici sistemici, rispetto al 2003, sono rimasti globalmente stabili, con una tendenza alla riduzione delle cefalosporine e dei macrolidi e ad un lieve incremento dei fluorochinoloni e delle penicilline [3].

Secondo una recente indagine comparativa condotta in diversi paesi europei e pubblicata su Lancet, il consumo medio pro capite di antimicrobici sistemici in Italia si colloca in una posizione medio-alta, circa $24 \mathrm{DDD} / 1000 \mathrm{abi}$ tanti/die, quando in Olanda è di sole 9 DDD/ 1000 abitanti/die, mentre in Francia raggiunge le 37 DDD/1000 abitanti/die [4].

Tuttavia, la differenza più rilevante che contraddistingue l'Italia dagli altri paesi europei nelle modalità di consumo degli antibiotici consiste nel maggior utilizzo della via parenterale rispetto alla via orale e, nell' ambito delle vie parenterali, nell'uso molto frequente della via intramuscolare rispetto alla via endovenosa. Queste differenze sono state costantemente osservate negli anni sia in ambito territoriale sia in ambito ospedaliero [5-8].

Negli anni ' 90 circa il $20 \%$ delle prescrizioni territoriali di antibiotici riguardava le forme iniettive. Nel 1997, ad esempio, i medici italiani extra-ospedalieri hanno effettuato oltre $53 \mathrm{mi}$ lioni di prescrizioni di antibiotici: poco meno del $18 \%$ di queste prescrizioni riguardavano antibiotici iniettivi scelti prevalentemente tra le cefalosporine (12,2\%) [7].

Il tipo e la sede dell'infezione e l'età del paziente sembrano condizionare i medici italiani nella scelta della via di somministrazione degli antibiotici. La via orale è preferita nelle infezioni delle alte vie respiratorie, nelle infezioni dell'orecchio e mastoide, nelle infezioni

\begin{tabular}{|c|c|c|c|}
\hline \multicolumn{2}{|c|}{$\begin{array}{l}\text { Nota AIFA } 55 \text { : } \\
\text { Antibiotici iniettabili per uso territoriale }\end{array}$} & \multicolumn{2}{|c|}{$\begin{array}{l}\text { Nota AIFA 56: } \\
\text { Antibiotici per continuità ospedale-territorio }\end{array}$} \\
\hline $\begin{array}{l}\text { cefamandolo } \\
\text { cefonicid } \\
\text { ceftezolo } \\
\text { cefurossima } \\
\text { cefmetazolo } \\
\text { cefotetan } \\
\text { cefoxitina } \\
\text { cefodizima } \\
\text { cefoperazone } \\
\text { cefotaxima } \\
\text { ceftazidima* } \\
\text { ceftizoxima } \\
\text { ceftriaxone } \\
\text { cefepime* }\end{array}$ & $\begin{array}{l}\text { mezlocillina } \\
\text { piperacillina } \\
\text { ampicillina + sulbactam } \\
\text { piperacillina + tazobactam* } \\
\text { ticarcillina + ac. clavulanico* } \\
\text { amikacina } \\
\text { gentamicina } \\
\text { netilmicina } \\
\text { tobramicina }\end{array}$ & $\begin{array}{l}\text { aztreonam } \\
\text { rifabutina } \\
\text { teicoplanina }\end{array}$ & $\begin{array}{l}\text { ertapenem } \\
\text { imipenem }+ \text { cilastatina } \\
\text { meropenem }\end{array}$ \\
\hline $\begin{array}{l}\text { La prescrizior } \\
\text { per l'uso com } \\
\text { 1. trattamento } \\
\text { vie urinarie, de } \\
\text { ginecologiche, } \\
\text { 2. trattamento } \\
\text { resistenti ai più } \\
\text { immunocompr }\end{array}$ & $\begin{array}{l}\text { del SSN degli antibiotici iniettabili } \\
\text { imitata alle seguenti condizioni: } \\
\text { ezioni gravi delle vie respiratorie, delle } \\
\text { intra-addominali, ostetrico- } \\
\text { olari; } \\
\text { infezioni causate da microrganismi } \\
\text { oiotici, particolarmente nei pazienti }\end{array}$ & \multicolumn{2}{|c|}{$\begin{array}{l}\text { La prescrizione a carico del SSN è limitata al } \\
\text { trattamento iniziato in ambito ospedaliero ed al } \\
\text { successivo utilizzo in ambito territoriale da parte del } \\
\text { Medico di Medicina Generale per garantire la } \\
\text { continuità terapeutica. }\end{array}$} \\
\hline $\begin{array}{l}\text { Un razionale u } \\
\text { l'ambiente territ } \\
\text { resistenze batte } \\
\text { ospedaliero ed } \\
\text { infezioni risolvik } \\
\text { Tali farmaci no } \\
\text { scelta terapeut } \\
\text { allo scopo di p } \\
\text { ciò vale in part } \\
\text { Pseudomonas } \\
\text { Per gli aminogl } \\
\text { associazione c } \\
\text { RSA o strutture } \\
\text { immunocompr } \\
\text { sottoposti a tra } \\
\text { scopo di poten }\end{array}$ & $\begin{array}{l}\text { tibiotici permette di preservare } \\
\text { spedaliero dalla diffusione delle } \\
\text { nendolo separato da quello } \\
\text { corso all'ospedalizzazione per trattare } \\
\text { ente al domicilio del paziente. } \\
\text { rappresentare, di norma, la prima } \\
\text { o riservati a casi selezionati, anche } \\
\text { rgere di ceppi resistenti sul territorio; } \\
\text { antibiotici impiegati nei confronti di } \\
\left.\text { contrassegnati da asterisco ( }{ }^{\star}\right) \text {. } \\
\text { ticolare è indicato l'impiego in } \\
\text { mine, in pazienti anziani che vivono in } \\
\text { bazienti defedati o } \\
\text { entemente dimessi dall'ospedale e/o } \\
\text { ratti con antibiotici a largo spettro, allo } \\
\text { are lo spettro d'azione antibatterica. }\end{array}$ & \multicolumn{2}{|c|}{$\begin{array}{l}\text { La prescrivibilità esclusiva in ambito ospedaliero è finalizzata } \\
\text { al mantenimento dell'efficacia ed alla contemporanea } \\
\text { prevenzione dell'insorgenza di resistenza batterica ai principi } \\
\text { attivi. } \\
\text { La scelta di iniziare un trattamento ospedaliero con tali } \\
\text { farmaci dovrebbe essere riservata alle infezioni gravi e in } \\
\text { assenza di alternative terapeutiche. } \\
\text { Ciò non impedisce, tuttavia, dopo la diagnosi e l'inizio del } \\
\text { trattamento, il mantenimento della continuità assistenziale } \\
\text { ospedale-territorio a carico del SSN, ove fosse necessario } \\
\text { proseguire la terapia a domicilio. }\end{array}$} \\
\hline
\end{tabular}

Tabella I

Sinossi delle Note AIFA 55 e 56 (revisione 2004) limitative della prescrizione degli antibiotici iniettabili a carico del SSN da parte del medico di Medicina Generale [1] 
delle vie genito-urinarie e del cavo orale, mentre la via parenterale è preferita nelle infezioni delle basse vie respiratorie e in una varietà di altre infezioni. Gli adulti e gli anziani sono i maggiori fruitori degli antibiotici iniettabili, mentre in età pediatrica la via parenterale viene usata raramente e solo nei casi strettamente indispensabili [7].

Le modalità di utilizzo degli antibiotici in Italia hanno sollevato critiche e numerose polemiche. In particolare, il confronto con gli Stati Uniti e con altri paesi europei di cultura anglosassone, spesso presi come modelli di riferimento sia scientifico sia comportamentale, ha indotto molti a criticare la presunta iperprescrizione domiciliare di antibiotici iniettabili, soprattutto cefalosporine di $\mathrm{III}^{\circ}$ generazione, ed ha spinto infine le Autorità Regolatorie ad adottare le Note AIFA limitative sopra citate.

Non c'è dubbio che vi fosse e ancora vi sia una qualche opportunità di ridimensionare l'uso di antibiotici parenterali in Italia, sostituendoli, ove possibile, con quelli orali. Occorre, tuttavia, essere molto cauti nel trarre conclusioni dai soli dati di prescrizione. Infatti, non esiste alcuna dimostrazione della superiorità dei risultati sanitari ottenuti nei paesi che adottano preferenzialmente la via orale rispetto a quelli ottenuti in Italia. Al contrario, semmai, vi sono indicazioni di risultati sanitari complessivamente inferiori a quelli ottenuti in Italia per quei paesi, come il Regno Unito, dove, soprattutto a domicilio, sono prescritti quasi esclusivamente antibiotici orali.

Un'indagine comparativa, condotta trasversalmente in diversi paesi europei, sulle modalità di gestione delle infezioni delle basse vie respiratorie, ha evidenziato che il tasso di ospedalizzazione nel Regno Unito è del 9\% contro il 2,8\% dell'Italia [8]. Inoltre, l'incidenza di morti per malattie dell' apparato respiratorio nel Regno Unito è del 15\% contro il 5,8\% registrato in Italia. Infine, alcuni illustri microbiologi clinici italiani sostengono che i tassi relativamente bassi di resistenze agli antibiotici registrati finora in Italia, comparativamente ad altri paesi europei anche confinanti, possa essere attribuito, almeno in parte, all'elevato consumo di antibiotici iniettabili.

L'introduzione delle note CUF 55, 55bis (ora unificate e riviste nella nota AIFA 55) e 56 ha indubbiamente ridotto il consumo degli antibiotici iniettabili in ambito extra-ospedaliero ed ha comportato una maggiore attenzione da parte della classe medica nell'usare razionalmente gli antibiotici. Non è dato sapere, tuttavia, quali conseguenze abbia avuto questa strategia limitativa sulla spesa ospedaliera e su altri costi sanitari o sociali correlabili alle infezioni acquisite a domicilio.
L'uso di antibiotici parenterali a domicilio deve essere considerato non solo come alternativa all'uso di antibiotici orali, ma anche e soprattutto come alternativa al ricovero e come opportunità di ridurre la durata della degenza ospedaliera per quei pazienti che, avendo necessità di un trattamento antibiotico parenterale, possono attuarlo totalmente o continuarlo al proprio domicilio.

In questo lavoro riconsideriamo quali siano i principali criteri microbiologici, clinici e farmacoeconomici sui quali si fonda la razionalità e la convenienza di una terapia antibiotica iniettiva domiciliare (TAID).

\section{CRITERI EPIDEMIOLOGICI}

Il fenomeno dell'insorgenza e del diffondersi delle resistenze batteriche agli antibiotici è correlato in modo complesso all'intensità e alla modalità d'uso degli antibiotici stessi, e alle condizioni dell' ambiente, capaci di favorirne o impedirne l'evoluzione. Gli ospedali e in particolare alcuni reparti ospedalieri, come le Unità di Terapia Intensiva, per la loro organizzazione assistenziale, la tipologia dei pazienti e l'intensità delle cure, sono sistemi noti per favorire l'insorgenza e la diffusione di resistenze batteriche problematiche, spesso con evoluzione di tipo epidemico-endemico.

Le infezioni acquisite in ospedale (nosocomiali) sono considerate un onere sanitario ed economico elevato per il SSN e la società, non solo per la loro elevata incidenza (circa 8-10\% dei ricoveri negli ospedali italiani), ma anche per l'alto rischio di mortalità e per la difficoltà delle cure a causa delle frequenti resistenze agli antibiotici disponibili. Riservare gli antibiotici iniettivi più potenti all'ambito ospedaliero sembra, dunque, razionale in considerazione della necessità di preservare strumenti essenziali per la cura delle infezioni nosocomiali più difficili. D'altra parte, si riteneva che in ambito extra-ospedaliero, ed in particolare in ambito domiciliare, circolassero patogeni e ceppi di patogeni differenti da quelli ospedalieri e che il problema delle resistenze fosse meno diffuso e problematico. Pertanto, molti autori ritenevano e ancora ritengono opportuno limitare l'uso degli antibiotici iniettabili in ambito extra-ospedaliero, in quanto non necessari o addirittura irrazionali, per evitare l'insorgenza e la circolazione di ceppi resistenti anche sul territorio. Queste posizioni sembrano, oggi, essere messe in crisi da una serie crescente di studi microbiologici che da qualche tempo evidenziano l'aumento costante di ceppi resistenti responsabili di infezioni acquisite a domicilio, segno evidente che ceppi un 
tempo confinati negli ospedali sono "fuoriusciti" da tali ambiti e si sono diffusi in intere aree geografiche.

Fin dagli anni' 70 era stato dimostrato come ceppi di $S$. aureus produttori di penicillinasi, resistenti alla penicillina ma sensibili alla meticillina, avevano raggiunto quote percentuali di prevalenza in ambito territoriale simili a quelle che da anni erano registrate solo negli ospedali (vedi figura 1) [9].

Nelle ultime due decadi, in stretta analogia con l'evoluzione delle resistenze mediate dalle penicillinasi, ceppi di S. aureus meticillino-resistenti (MRSA), un tempo tipicamente confinati negli ospedali e nelle strutture sanitarie per lungodegenti, sono stati isolati con crescente frequenza anche in ambito territoriale [10-17]. In realtà, l'origine di questi ceppi MRSA circolanti sul territorio non è chiara ed è oggetto di discussione. In ogni caso vi è un sostanziale accordo nel ritenere che questo fenomeno, destinato ancora a crescere, sia dovuto alla pressione selettiva che favorisce la selezione dei ceppi resistenti sia in ambito ospedaliero che territoriale [9].

Alcuni studi indicherebbero un aumento di portatori di ceppi MRSA tra coloro che non hanno fattori di rischio sanitari noti. Una metaanalisi, pubblicata recentemente, ha preso in esame sia gli studi che riportavano la prevalenza di ceppi MRSA acquisiti in ambito extraospedaliero tra i ceppi MRSA isolati da pazienti ricoverati, sia gli studi che riportavano la prevalenza di colonizzazione in soggetti non ospedalizzati. I risultati sono riassunti nella tabella II [18].

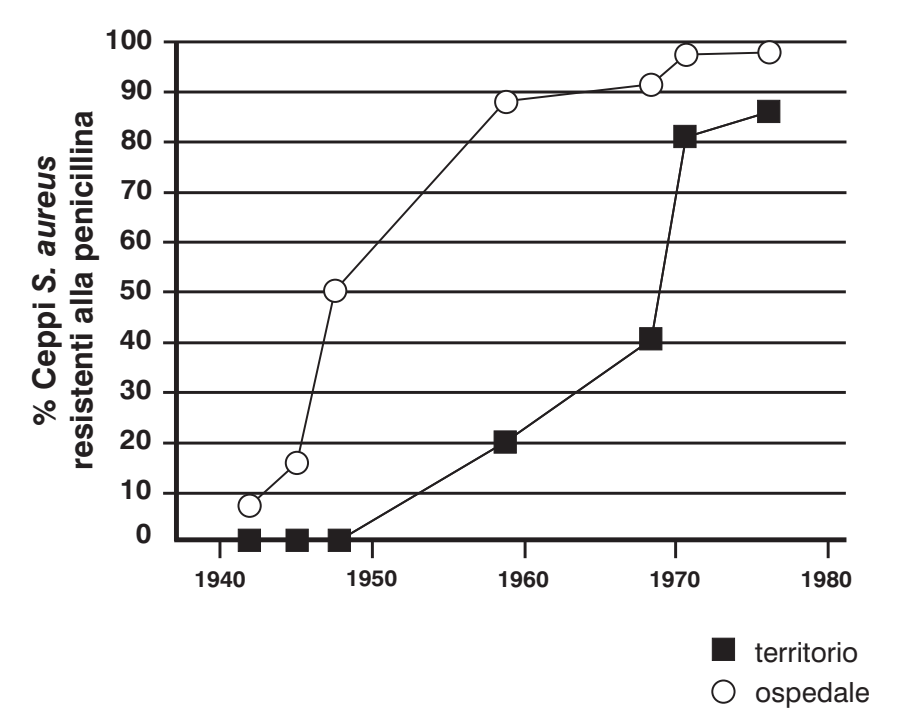

Figura 1

Evoluzione temporale della prevalenza di ceppi di Staphylococcus aureus, produttori di penicillinasi, resistenti alla penicillina, meticillino-sensibili, isolati in ambito ospedaliero ed in ambito extra-ospedaliero (modificata da: Chambers $H F, 2001)$ [9]
La meta-analisi condotta su 27 studi retrospettivi e su 5 studi prospettici ha indicato rispettivamente nel $30,2 \%$ e nel $37,3 \%$ la prevalenza di ceppi MRSA acquisiti a domicilio tra i ceppi MRSA isolati da pazienti ricoverati. L'85\% di tutti i pazienti con MRSA acquisiti a domicilio presentavano uno o più fattori di rischio associati all' assistenza sanitaria. La frequenza di colonizzazioni MRSA tra i soggetti non ospedalizzati variava molto in funzione della tipologia dei soggetti considerati nei vari studi. La meta-analisi condotta su un pool di 10 studi ha indicato in una frequenza media dell'1,3\% (Intervallo di Confidenza 95\%: 1,04\%$1,53 \%$ ) la prevalenza di colonizzazioni MRSA tra i soggetti non ospedalizzati. Il rischio di essere portatori sani è superiore nei soggetti che vivono o lavorano in strutture sanitarie rispetto a coloro che non hanno contatti con strutture sanitarie ( $R R=2,35$, CI 95\%: 1,56-3,53). La maggioranza dei pazienti o dei portatori di MRSA acquisita in ambito domiciliare presenta uno o più fattori di rischio (recente ricovero in ospedale, recente visita a ricoverati, recente assegnazione a programmi di ospedalizzazione domiciliare o di assistenza domiciliare integrata, recente esposizione ad antibiotici, presenza di patologie croniche, uso di farmaci parenterali, stretto contatto con una persona a rischio di MRSA). A conferma di ciò, la prevalenza di MRSA è molto bassa $(0,2 \%)$ tra coloro che non hanno o non hanno avuto contatto con strutture sanitarie [18].

Da questa meta-analisi si ricava una chiara conferma dell'ipotesi che occorra controllare la trasmissione nosocomiale di ceppi MRSA per attuare un efficace controllo della loro diffusione sul territorio.

Gli stafilococchi, in particolare lo S. aureus, rappresentano una delle principali cause delle infezioni acquisite sia in ospedale sia a domicilio. Nel nostro Paese, le infezioni da stafilococchi meticillino- resistenti rappresentano oltre il $50 \%$ delle infezioni dovute a stafilococco aureo e oltre il $60 \%$ delle infezioni da stafilococchi coagulasi-negativi gestite in ospedale; tuttavia ceppi di stafilococchi meticillino-resistenti cominciano a essere segnalati anche a livello territoriale [17].

Il programma di sorveglianza microbiologica SENTRY ha evidenziato il ruolo preminente dello $S$. aureus come agente eziologico delle batteriemie, delle polmoniti e delle infezioni della cute e dei tessuti molli [19]. A livello domiciliare lo $S$. aureus è frequentemente implicato nelle infezioni della basse vie respiratorie (LRTI) degli anziani [20].

Due sistemi internazionali di sorveglianza microbiologica, l'Alexander Project e il PROTEKT Study, e programmi collaborativi 


\begin{tabular}{lc}
\hline \multicolumn{1}{c}{ Modalità degli studi e origine dei ceppi di isolamento } & $\begin{array}{c}\text { Prevalenza } \\
(\mathbf{C l ~ 9 5 \% )}\end{array}$ \\
\hline Ceppi isolati da pazienti ospedalizzati & \\
Meta-analisi su 27 studi retrospettivi & $30,2 \%$ \\
Meta-analisi su 5 studi prospettici & $37,3 \%$ \\
$\begin{array}{l}\text { Ceppi isolati da portatori in ambito extra-ospedaliero } \\
\begin{array}{l}\text { Meta-analisi su } 10 \text { studi }(n=8350) \text { basati sulla sorveglianza } \\
\text { microbiologica territoriale }\end{array}\end{array}$ \\
$\begin{array}{l}\text { Meta-analisi su } 4 \text { studi ( } n=191) \text { condotti su familiari a contatto con } \\
\text { pazienti MRSA dimessi dall'ospedale }\end{array}$ \\
$\begin{array}{l}\text { Meta-analisi su } 4 \text { studi }(n=517) \text { condotti su compagni di squadra o } \\
\text { collaboratori di soggetti colonizzati da MRSA }\end{array}$ \\
\hline
\end{tabular}

nazionali di sorveglianza, hanno evidenziato in questi ultimi anni l'emergere e il diffondersi in ambito extra-ospedaliero di numerose resistenze agli antibiotici in tutti i principali patogeni responsabili delle infezioni respiratorie [21-26].

La prevalenza delle resistenze dello $S$. pneumoniae, dello $S$. pyogenes, dell' $H$. influenzae e della $M$. catarrhalis e dello $S$. aureus variano molto in relazione alla classe di antibiotico considerata e alla regione geografica.

Nei paesi dell'Europa Occidentale la prevalenza dei ceppi di pneumococco penicillinoresistenti è mediamente ormai del $25-30 \%$, ma con un'elevata differenza tra i diversi paesi, anche confinanti, e variazioni che vanno da $<5 \%$ in Olanda a $>50 \%$ in Francia [26]. In Italia la prevalenza delle resistenze dello pneumococco alla penicillina è intorno al 10\% [27].

Durante gli anni ' 90 in Europa è stato registrato un rapido aumento delle resistenze ai macrolidi da parte di ceppi di pneumococco sia penicillino-sensibili che penicillino-resistenti, con prevalenze che hanno raggiunto il $57 \%$ in Francia e dal $32 \%$ al $43 \%$ in Italia. Nella maggior parte dei paesi europei lo sviluppo della resistenza ai macrolidi dello pneumococco è stato parallelo a quello della resistenza alla penicillina. In Italia, invece, vi è stata una netta dissociazione con aumenti delle resistenze ai macrolidi da $<5 \%$ a $>40 \%$, mentre le resistenze alla penicillina restavano stabili attorno al $10 \%[26,27]$.

Uno studio recente condotto dall'European Antimicrobial Resistance Surveillance System (EARSS) ha prospettato una stretta correlazione tra lo sviluppo delle resistenze alla penicillina dello pneumococco e volumi di vendita territoriale di beta-lattamine e di macrolidi [28].

Tuttavia, secondo autorevoli ricercatori, la dissociazione, osservata in Italia con lo pneumococco, tra prevalenza di resistenze ai macrolidi e prevalenza di resistenze alla penicilli- na sarebbe correlata positivamente sia all'ampio uso di cefalosporine e beta-lattamine parenterali, che differenzia l'Italia dagli altri paesi europei, sia all' ampia gamma di classi di antibiotici utilizzati, compresi i macrolidi orali $[28,30]$.

Pur riconoscendo una correlazione diretta tra quantità di antibiotici prescritti sul territorio e prevalenza di resistenze agli stessi, l'elevato uso di cefalosporine e beta-lattamine iniettive comporterebbe una riduzione del rischio di indurre selezione di ceppi patogeni perché la somministrazione parenterale induce concentrazioni plasmatiche e tissutali più elevate capaci di eradicare rapidamente i patogeni sensibili, limitando la pressione selettiva [31].

Nei paesi europei, soprattutto in quelli del bacino mediterraneo, sono elevate e ancora in aumento le prevalenze di resistenze nei patogeni produttori di beta-lattamasi: mediamente 1 ' $11-19 \%$ dei ceppi di $H$. influenzae e 1'85-100\% dei ceppi di M. catarrhalis. In Italia i ceppi di $H$. influenzae produttori di betalattamasi sono sensibilmente meno numerosi e oscillano dal $2 \%$ al $8 \%$ degli isolati $[26,30]$.

In anni recenti, in molti ospedali in tutto il mondo, è aumentata la prevalenza di ceppi di Enterobacteriaceae produttori di betalattamasi a spettro esteso (ESBL). Dagli ospedali questi ceppi sono stati disseminati anche sul territorio, creando gravi problemi nella terapia empirica delle infezioni acquisite a domicilio. Un'indagine microbiologica condotta nel Sud Israele ha evidenziato che il 5\% dei ceppi di Enterobacteriaceae ESBL-positivi isolati da emoculture erano di origine extra-ospedaliera [32].

Un'indagine microbiologica, condotta in Italia nel 1999, ha evidenziato che il 6,3\% degli oltre 8.000 ceppi di Enterobacteriaceae d'isolamento erano ESBL positivi. Tra questi, il 73,6\% era rappresentato da ceppi di Klebsiella pneumoniae, Proteus mirabilis ed Escherichia coli. Tutti i ceppi ESBL-positivi erano rimasti sensibili ai carbapenemi, mentre la sensibilità ad altri antibiotici variava dal $91 \%$
Tabella II

Risultati di meta-analisi degli studi che riportano la prevalenza di MRSA acquisita in ambito extra-ospedaliero, suddivisi in funzione delle modalità degli studi pubblicati dal 1966 al 2002 (modificata da: Salgado CD et al, 2003) [18] 
per la piperacillina/tazobactam, 1'85\% per l'amoxicillina/clavulanato, il $78 \%$ per la cefoxitina, il $76 \%$ per l'amikacina, il $61 \%$ per l'ampicillina/sulbactam, il $58 \%$ per la ciprofloxacina e il $56 \%$ per la gentamicina [33].

Da una successiva sorveglianza nazionale condotta in Italia nel 2003 su 8681 isolati di Enterobacteriaceae di provenienza sia ospedaliera che comunitaria, la prevalenza di ceppi ESBL positivi è stata del 7,4\% in isolati di origine ospedaliera (range 4,8-12,6\%) e 3,5\% (range $0,8-5,6 \%$ ) in quelli di origine comunitaria [34]. Questi dati confermano che la diffusione di tale tipo di resistenza batterica non è più confinata solo in ospedale e pertanto la presenza di ceppi produttori di ESBL deve essere tenuta in considerazione quando si sceglie l'antibiotico per il trattamento delle infezioni al domicilio del paziente.

L'uso allargato e soprattutto il cattivo uso o uso irrazionale di cefalosporine di $\mathrm{III}^{\circ}$ generazione sono considerati cause favorenti l'insorgenza e il diffondersi di ceppi produttori di ESBL. Tale meccanismo di resistenza si può attivare anche nel corso di un singolo trattamento [35,36].

Le urinocolture di pazienti ambulatoriali sono un'utile fonte di informazione per sorvegliare le resistenze agli antibiotici dei patogeni circolanti in ambito extra-ospedaliero. Nella tabella III sono riportate le percentuali dei principali batteri patogeni isolati dai campioni di urine di pazienti ambulatoriali afferenti ad un ospedale regionale di riferimento (Molinette di Torino) negli anni 2000-2003. La tabella IV riporta le percentuali di sensibilità dei patogeni rilevanti ai principali antibiotici testati [37].

Salvo poche eccezioni, in questa casistica le percentuali di sensibilità agli antibiotici appaiono sostanzialmente stabili negli ultimi anni. Tra gli antibiotici testati, la piperacillina/ tazobactam dimostra di aver mantenuto un'elevata percentuale di attività nei confronti dei ceppi di E. coli, K. Pneumoniae, P. mirabilis e $S$. aureus; inoltre negli ultimi anni ha progressivamente recuperato l'attività nei confronti anche di $P$. aeruginosa. L'attività antibatterica di piperacillina/tazobactam è sensibilmente superiore a quella di amoxicillina/clavulanato, ceftriaxone, gentamicina, ciprofloxacina, mentre è simile o leggermente inferiore solo a quella di imipenem [37].

Questi risultati sono analoghi a quelli ottenuti su una raccolta di patogeni isolati da pazienti anziani affetti da infezioni delle basse vie respiratorie nel corso dello studio DEDALO [20]. Le percentuali dei vari patogeni isolati da broncoaspirati o da escreati di pazienti affetti da CAP e da AECOPD sono riportate nella tabella $\mathrm{V}$.

In questa casistica di pazienti anziani con infezioni delle basse vie respiratorie acquisite a domicilio vi è una percentuale relativamente elevata di $S$. aureus e di $P$. aeruginosa. Inoltre, il $40,2 \%$ dei ceppi di Enterobacteriaceae, il 40,4\% degli altri Gram-negativi e il 69,8\% dei Gram-positivi sono batteri produttori di betalattamasi [20].

Nella tabella VI sono riportate le percentuali dei principali patogeni isolati in questa casistica e per ognuno dei patogeni le percentuali di sensibilità ai sei antibiotici testati, scelti sulla base della loro frequente utilizzazione anche in terapie domiciliari. Infine, per ogni antibiotico sono calcolati i tassi teorici di eradicazione (successo microbiologico), assumendo che la distribuzione delle sensibilità sui germi non indicati sia proporzionale a quella dei germi testati, che rappresentano oltre il $75 \%$ del totale.

Questa stima indica che l'utilizzo della piperacillina/tazobactam nel trattamento delle
Tabella III

Distribuzione

percentuale dei

patogeni isolati da

campioni di urine di

pazienti extra-

ospedalieri afferenti al

laboratorio di

microbiologia

dell'ospedale di

riferimento regionale.

(dati da file personali R.

Serra) [37]

\begin{tabular}{lcccc}
\hline \multicolumn{1}{c}{ Anno } & $\mathbf{2 0 0 0}$ & $\mathbf{2 0 0 1}$ & $\mathbf{2 0 0 2}$ & $\mathbf{2 0 0 3}$ (I'sem) \\
\hline Escherichia coli & $56,57 \%$ & $55,06 \%$ & $54,62 \%$ & $61,96 \%$ \\
Enterococcus faecalis & $11,43 \%$ & $11,79 \%$ & $9,73 \%$ & $7,52 \%$ \\
Klebsiella pneumoniae & $3,89 \%$ & $4,00 \%$ & $4,72 \%$ & $4,56 \%$ \\
Proteus mirabilis & $3,43 \%$ & $2,45 \%$ & $3,85 \%$ & $3,19 \%$ \\
Pseudomonas aeruginosa & $3,20 \%$ & $4,34 \%$ & $3,28 \%$ & $2,28 \%$ \\
Staphylococcus aureus & $1,37 \%$ & $1,78 \%$ & $1,64 \%$ & $1,37 \%$ \\
Totale isolati & $\mathbf{1 0 0 , 0 0 \%}$ & $\mathbf{1 0 0 , 0 0 \%}$ & $\mathbf{1 0 0 , 0 0 \%}$ & $\mathbf{1 0 0 , 0 0 \%}$ \\
\hline
\end{tabular}




\begin{tabular}{|c|c|c|c|c|}
\hline Anno & 2000 & 2001 & 2002 & 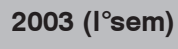 \\
\hline \multicolumn{5}{|l|}{ Amoxicillina } \\
\hline Escherichia coli & $68,6 \%$ & $47,5 \%$ & $57,6 \%$ & $65,4 \%$ \\
\hline Enterococcus faecalis & $100,0 \%$ & $99,0 \%$ & $100,0 \%$ & $100,0 \%$ \\
\hline Proteus mirabilis & $66,7 \%$ & $47,6 \%$ & $51,3 \%$ & $28,6 \%$ \\
\hline Streptococcus agalactiae & $100,0 \%$ & $100,0 \%$ & $100,0 \%$ & $100,0 \%$ \\
\hline \multicolumn{5}{|l|}{ Amoxicillina/clavulanico } \\
\hline Escherichia coli & $91,7 \%$ & $91,5 \%$ & $92,0 \%$ & $96,0 \%$ \\
\hline Klebsiella pneumoniae & $87,9 \%$ & $91,4 \%$ & $98,0 \%$ & $95,0 \%$ \\
\hline Proteus mirabilis & $92,6 \%$ & $90,5 \%$ & $97,4 \%$ & $92,9 \%$ \\
\hline Staphylococcus aureus & $50,0 \%$ & $20,0 \%$ & $64,7 \%$ & $66,7 \%$ \\
\hline \multicolumn{5}{|l|}{ Piperacillina/tazobactam } \\
\hline Escherichia coli & $100,0 \%$ & $97,0 \%$ & $95,4 \%$ & $98,9 \%$ \\
\hline Klebsiella pneumoniae & $100,0 \%$ & $90,9 \%$ & $95,9 \%$ & $95,0 \%$ \\
\hline Proteus mirabilis & $100,0 \%$ & $100,0 \%$ & $100,0 \%$ & $100,0 \%$ \\
\hline Pseudomonas aeruginosa & $50,0 \%$ & $73,0 \%$ & $90,9 \%$ & $80,0 \%$ \\
\hline Staphylococcus aureus & $100,0 \%$ & $100,0 \%$ & $100,0 \%$ & $100,0 \%$ \\
\hline \multicolumn{5}{|l|}{ Cefazolina } \\
\hline Escherichia coli & $93,8 \%$ & $44,2 \%$ & $59,6 \%$ & $70,6 \%$ \\
\hline Klebsiella pneumoniae & $87,9 \%$ & $73,5 \%$ & $83,3 \%$ & $80,0 \%$ \\
\hline Proteus mirabilis & $81,5 \%$ & $76,2 \%$ & $73,7 \%$ & $57,1 \%$ \\
\hline Staphylococcus aureus & $60,0 \%$ & $23,1 \%$ & $64,7 \%$ & $66,7 \%$ \\
\hline \multicolumn{5}{|l|}{ Cefuroxime } \\
\hline Escherichia coli & $95,9 \%$ & $89,8 \%$ & $94,6 \%$ & $90,4 \%$ \\
\hline Klebsiella pneumoniae & $90,6 \%$ & $84,4 \%$ & $97,8 \%$ & $88,9 \%$ \\
\hline Proteus mirabilis & $100,0 \%$ & $85,7 \%$ & $84,6 \%$ & $78,6 \%$ \\
\hline \multicolumn{5}{|l|}{ Ceftriaxone } \\
\hline Escherichia coli & $99,6 \%$ & $99,2 \%$ & $99,6 \%$ & $99,6 \%$ \\
\hline Klebsiella pneumoniae & $100,0 \%$ & $100,0 \%$ & $100,0 \%$ & $100,0 \%$ \\
\hline Proteus mirabilis & $96,3 \%$ & $90,5 \%$ & $87,2 \%$ & $78,6 \%$ \\
\hline Pseudomonas aeruginosa & $50,0 \%$ & $47,4 \%$ & $48,5 \%$ & $10,0 \%$ \\
\hline \multicolumn{5}{|l|}{ Ceftazidime } \\
\hline Escherichia coli & $99,4 \%$ & $99,8 \%$ & $99,5 \%$ & $99,6 \%$ \\
\hline Klebsiella pneumoniae & $100,0 \%$ & $100,0 \%$ & $100,0 \%$ & $100,0 \%$ \\
\hline Proteus mirabilis & $92,6 \%$ & $90,5 \%$ & $87,2 \%$ & $92,9 \%$ \\
\hline Pseudomonas aeruginosa & $89,3 \%$ & $89,5 \%$ & $84,8 \%$ & $70,0 \%$ \\
\hline \multicolumn{5}{|l|}{ Imipenem } \\
\hline Escherichia coli & $99,8 \%$ & $99,8 \%$ & $99,8 \%$ & $100,0 \%$ \\
\hline Klebsiella pneumoniae & $100,0 \%$ & $100,0 \%$ & $100,0 \%$ & $100,0 \%$ \\
\hline Proteus mirabilis & $100,0 \%$ & $100,0 \%$ & $100,0 \%$ & $100,0 \%$ \\
\hline Pseudomonas aeruginosa & $78,6 \%$ & $92,1 \%$ & $84,8 \%$ & $70,0 \%$ \\
\hline \multicolumn{5}{|l|}{ Ciprofloxacina } \\
\hline Escherichia coli & $86,1 \%$ & $83,7 \%$ & $84,0 \%$ & $84,6 \%$ \\
\hline Enterococcus faecalis & $79,8 \%$ & $83,7 \%$ & $68,0 \%$ & $64,7 \%$ \\
\hline Klebsiella pneumoniae & $100,0 \%$ & $97,1 \%$ & $98,0 \%$ & $88,9 \%$ \\
\hline Proteus mirabilis & $77,8 \%$ & $71,4 \%$ & $76,9 \%$ & $85,7 \%$ \\
\hline Pseudomonas aeruginosa & $50,0 \%$ & $55,3 \%$ & $51,3 \%$ & $40,0 \%$ \\
\hline Staphylococcus aureus & $25,0 \%$ & $20,0 \%$ & $47,1 \%$ & $66,7 \%$ \\
\hline \multicolumn{5}{|l|}{ Gentamicina } \\
\hline Escherichia coli & $92,5 \%$ & $92,1 \%$ & $92,4 \%$ & $97,8 \%$ \\
\hline Klebsiella pneumoniae & $100,0 \%$ & $100,0 \%$ & $100,0 \%$ & $100,0 \%$ \\
\hline Proteus mirabilis & $77,8 \%$ & $66,7 \%$ & $59,0 \%$ & $78,6 \%$ \\
\hline Pseudomonas aeruginosa & $57,1 \%$ & $50,0 \%$ & $66,7 \%$ & $60,0 \%$ \\
\hline Staphylococcus aureus & $45,5 \%$ & $26,7 \%$ & $56,3 \%$ & $66,7 \%$ \\
\hline \multicolumn{5}{|l|}{ Cotrimoxazolo } \\
\hline Escherichia coli & $81,2 \%$ & $77,1 \%$ & $78,5 \%$ & $79,4 \%$ \\
\hline Klebsiella pneumoniae & $93,9 \%$ & $88,6 \%$ & $93,9 \%$ & $100,0 \%$ \\
\hline Proteus mirabilis & $63,0 \%$ & $57,1 \%$ & $64,1 \%$ & $50,0 \%$ \\
\hline Staphylococcus aureus & $91,7 \%$ & $100,0 \%$ & $100,0 \%$ & $100,0 \%$ \\
\hline \multicolumn{5}{|l|}{ Vancomicina } \\
\hline Enterococcus faecalis & $99,0 \%$ & $100,0 \%$ & $100,0 \%$ & $100,0 \%$ \\
\hline Staphylococcus aureus & $100,0 \%$ & $93,3 \%$ & $100,0 \%$ & $100,0 \%$ \\
\hline
\end{tabular}

Tabella IV

Percentuali di sensibilità ai principali antibiotici dei patogeni più frequentemente isolati da urinocolture di pazienti ambulatoriali afferenti al laboratorio di microbiologia clinica di un ospedale di riferimento regionale. (dati da file personali R. Serra) [37] 
Tabella $V$

Batteri patogeni isolati da pazienti anziani con CAP e AECOPD

(modificata da: Sanguinetti $C M$ et al, 2000) [20]

\begin{tabular}{lccc}
\hline & CAP & AECOPD & Totale LRTI \\
\hline$N^{\circ}$ pazienti testati & 187 & 877 & 1064 \\
$N^{\circ}$ pazienti con coltura positiva su broncoaspirato & $31 / 60$ & $69 / 89$ & $100 / 149$ \\
$N^{\circ}$ pazienti con coltura positiva su sputo & $108 / 127$ & $627 / 788$ & $735 / 915$ \\
$N^{\circ}$ complessivo di pazienti con coltura positiva & $139 / 187$ & $706 / 877$ & $835 / 1064$ \\
\% pazienti con coltura positiva & $74,3 \%$ & $80,5 \%$ & $78,5 \%$ \\
& & Colture Positive (\%) & \\
E. coli & $4,1 \%$ & $4,5 \%$ & $4,4 \%$ \\
Enterobacter spp & $2,0 \%$ & $4,7 \%$ & $4,3 \%$ \\
H. influenzae & $11,2 \%$ & $14,1 \%$ & $13,6 \%$ \\
K. pneumoniae & $9,2 \%$ & $2,1 \%$ & $3,2 \%$ \\
M. catarrhalis & $3,1 \%$ & $4,8 \%$ & $4,5 \%$ \\
P. aeruginosa & $16,3 \%$ & $20,5 \%$ & $19,8 \%$ \\
S. aureus & $12,2 \%$ & $11,8 \%$ & $11,9 \%$ \\
S. pneumoniae & $13,3 \%$ & $9,7 \%$ & $10,3 \%$ \\
Serratia spp & $4,1 \%$ & $3,5 \%$ & $3,6 \%$ \\
Subtotale & $75,5 \%$ & $75,7 \%$ & $75,7 \%$ \\
\hline
\end{tabular}

infezioni delle basse vie respiratorie dei pazienti anziani ha una probabilità di successo sensibilmente maggiore, rispetto a quella di cefepime, ceftazidime e ciprofloxacina, e nettamente superiore a quella di amoxicillina e ceftriaxone.

In conclusione, l'epidemiologia microbiologica indica che sono in aumento, anche in Italia, le infezioni acquisite a domicilio sostenute da patogeni resistenti ai comuni antibiotici orali e a diversi antibiotici parenterali. Questo risultato è correlabile sia con l'ampio uso di antibiotici in ambito ospedaliero ed extraospedaliero sia con la diffusione sul territorio di ceppi patogeni resistenti, un tempo confinati solo negli ospedali. Vi sono ragionevoli argomenti per sostenere che la somministrazione parenterale di antibiotici, in confronto con quella orale, riduce la pressione selettiva e limita quindi l'insorgenza di nuove resistenze. D'altra parte, le più recenti indagini epidemiologiche e l'aumento delle resistenze sul territorio evidenziano l'opportunità, anzi la necessità, di utilizzare antibiotici iniettivi, fino-

\begin{tabular}{|c|c|c|c|c|c|c|c|c|c|c|c|c|c|}
\hline \multicolumn{2}{|c|}{ Colture Positive in LRTI } & \multicolumn{2}{|c|}{ AMOX } & \multicolumn{2}{|c|}{ CEFEP } & \multicolumn{2}{|c|}{ CEFTAZ } & \multicolumn{2}{|c|}{ CEFTRX } & \multicolumn{2}{|c|}{ CIPRO } & \multicolumn{2}{|c|}{ PIP/TAZO } \\
\hline Principali Patogeni & $\begin{array}{c}\% \\
\text { isolati }\end{array}$ & $\begin{array}{c}\% \\
\text { sens }\end{array}$ & $\begin{array}{c}\% \\
\text { succ }\end{array}$ & $\begin{array}{l}\% \\
\text { sens }\end{array}$ & $\begin{array}{c}\% \\
\text { succ }\end{array}$ & $\begin{array}{c}\% \\
\text { sens }\end{array}$ & $\begin{array}{c}\% \\
\text { succ }\end{array}$ & $\begin{array}{l}\% \\
\text { sens }\end{array}$ & $\begin{array}{c}\% \\
\text { succ }\end{array}$ & $\begin{array}{l}\% \\
\text { sens }\end{array}$ & $\begin{array}{c}\% \\
\text { succ }\end{array}$ & $\begin{array}{l}\% \\
\text { sens }\end{array}$ & $\begin{array}{c}\% \\
\text { succ }\end{array}$ \\
\hline E coli & 4,4 & 76,5 & 3,4 & 100,0 & 4,4 & 100,0 & 4,4 & 88,2 & 3,9 & 100,0 & 4,4 & 94,1 & 4,2 \\
\hline Enterobacter spp & 4,3 & 17,6 & 0,8 & 88,2 & 3,8 & 76,5 & 3,3 & 76,5 & 3,3 & 88,2 & 3,8 & 82,4 & 3,5 \\
\hline $\mathrm{H}$ influenzae & 13,6 & 95,8 & 13,1 & 91,7 & 12,5 & 95,8 & 13,1 & 93,8 & 12,8 & 93,8 & 12,8 & 95,8 & 13,1 \\
\hline K pneumoniae & 3,2 & 75,0 & 2,4 & 93,8 & 3,0 & 100,0 & 3,2 & 100,0 & 3,2 & 93,8 & 3,0 & 100,0 & 3,2 \\
\hline M catarrhalis & 4,5 & 88,9 & 4,0 & 94,4 & 4,3 & 100,0 & 4,5 & 94,4 & 4,3 & 94,4 & 4,3 & 100,0 & 4,5 \\
\hline$P$ aeruginosa & 19,8 & 6,3 & 1,2 & 63,3 & 12,6 & 65,8 & 13,0 & 27,8 & 5,5 & 72,2 & 14,3 & 74,7 & 14,8 \\
\hline S aureus & 11,9 & 62,2 & 7,4 & 64,9 & 7,7 & 62,2 & 7,4 & 62,2 & 7,4 & 56,8 & 6,7 & 64,9 & 7,7 \\
\hline S pneumoniae & 10,3 & 89,2 & 9,2 & 78,4 & 8,1 & 89,2 & 9,2 & 94,6 & 9,7 & 89,2 & 9,2 & 86,5 & 8,9 \\
\hline Serratia spp & 3,6 & 11,1 & 0,4 & 77,8 & 2,8 & 83,3 & 3,0 & 77,8 & 2,8 & 88,9 & 3,2 & 77,8 & 2,8 \\
\hline Subtotale & 75,7 & & 41,9 & & 59,1 & & 61,1 & & 52,9 & & 61,7 & & 62,7 \\
\hline $\begin{array}{l}\text { Totale successi } \\
\text { extrapolati al } 100 \%\end{array}$ & 100,0 & & 55,3 & & 78,1 & & 80,8 & & 69,9 & & 81,6 & & 82,9 \\
\hline
\end{tabular}

$\%$ succ $=$ percentuale teorica di successo calcolata considerando la \% di sensibilità di ciascun antibiotico rispetto alla \% di isolati di ciascuna specie

Tabella VI

Percentuali dei principali patogeni isolati da broncoaspirato o da escreato di pazienti anziani affetti da infezioni delle basse vie respiratorie acquisite a domicilio, percentuali di sensibilita a sei antibiotici comunemente utilizzati anche a domicilio e stima della percentuale teorica di successi microbiologici di ciascun antibiotico. (dati microbiologici da: Sanguinetti CM et al, 2000 mod.) [20] 
ra riservati ai pazienti ospedalizzati, per trattare empiricamente a domicilio le più comuni infezioni (es. polmoniti, infezioni complicate delle vie urinarie, ecc.) e sperare di ottenere un'elevata probabilità di successo. L'uso parenterale degli antibiotici a domicilio non è, dunque, da considerare in se stesso irrazionale, mentre può rappresentare il fattore determinante per ottenere il massimo beneficio sanitario, ridurre i rischi per il paziente e limitare l'insorgenza e la diffusione delle resistenze agli antibiotici.

\section{CRITERI CLINICI}

Ogni tipo di infezione presenta problemi specifici di diagnosi e cura che non possono essere affrontati e discussi in questa sede. Qui interessa solo evidenziare alcuni nodi decisionali che il medico curante di Medicina Genera- le deve affrontare e risolvere nella gestione di un paziente che si presenti con segni e sintomi riferibili ad una qualche infezione.

Dopo aver constatato il quadro clinico e avere formulato un'ipotesi diagnostica, il medico deve decidere se sia necessario il ricovero in ospedale oppure se il paziente possa essere gestito a domicilio senza rischi e particolari problemi. L'accesso all'ospedale può rendersi necessario sia per confermare la diagnosi sia per garantire un tipo di trattamento non effettuabile a domicilio.

Nel caso decida di non ricoverare il paziente, il medico deve successivamente scegliere quale antibiotico o associazione di antibiotici prescrivere, quale via di somministrazione e quali posologie utilizzare. Nell'operare queste scelte il medico dovrà considerare il profilo microbiologico e farmacologico degli antibiotici disponibili, il loro spettro d'azione, le per-

\begin{tabular}{|c|c|c|c|c|}
\hline Via & & Vantaggi & & Svantaggi \\
\hline Orale & $\begin{array}{l}0 \\
0 \\
0 \\
0 \\
0\end{array}$ & $\begin{array}{l}\text { Ottima accettabilità e miglior comfort } \\
\text { del paziente e familiari } \\
\text { Autogestibile dal paziente e familiari } \\
\text { Minima professionalità o } \\
\text { addestramento richiesti } \\
\text { Maggiore indipendenza e mobilità del } \\
\text { paziente } \\
\text { Nessun rischio di flebiti, infezioni, o } \\
\text { altre complicazioni da iniezione } \\
\text { Consente risparmi su: } \\
\text { - costo di acquisto degli antibiotici } \\
\text { - costo di somministrazione (materiali } \\
\text { e lavoro) } \\
\text { - giorni di degenza } \\
\text { - numero di ricoveri }\end{array}$ & प & $\begin{array}{l}\text { Minore controllo della compliance del } \\
\text { paziente } \\
\text { Maggior rischio di sottoesposizione } \\
\text { allantibiotico con conseguente: } \\
\text { - insuccesso terapeutico } \\
\text { - rischio di resistenze } \\
\text { Intolleranza e reazioni awerse } \\
\text { gastrointestinali } \\
\text { Impossibile nei pazienti con nausea e } \\
\text { vomito o altri disturbi del transito } \\
\text { esofageo }\end{array}$ \\
\hline Endovenosa & $\begin{array}{l}\text { 口 } \\
\text { 口 }\end{array}$ & $\begin{array}{l}\text { Biodisponibilità controllabile: } \\
\text { - tutta la dose disponibile } \\
\text { - velocità di infusione controllabile } \\
\text { - evitato il metabolismo di primo } \\
\text { passaggio } \\
\text { Possibile azione rapida e immediata } \\
\text { Minore dolore rispetto alla i.m. }\end{array}$ & $\begin{array}{l}\text { Q } \\
\text { Q } \\
\text { Q } \\
\text { Q } \\
\text { Q }\end{array}$ & $\begin{array}{l}\text { Maggiori rischi di reazioni awerse } \\
\text { dose-correlate } \\
\text { Maggiori rischi di reazioni allergiche } \\
\text { immediate } \\
\text { Lesioni endoteliali e flogosi } \\
\text { Contaminazione batterica } \\
\text { Contaminazione con eccipienti } \\
\text { Emboli gassosi } \\
\text { Necessario il ricovero o servizi } \\
\text { domiciliari integrati con la } \\
\text { partecipazione di medici } \\
\text { Maggiori costi per: } \\
\text { - acquisto farmaci } \\
\text { - materiali per la somministrazione } \\
\text { - lavoro del personale sanitario } \\
\text { qualificato }\end{array}$ \\
\hline Intramuscolare & $\begin{array}{l}\text { प } \\
\text { Q }\end{array}$ & $\begin{array}{l}\text { Biodisponibilità quasi totale } \\
\text { Evita i rischi da picco di } \\
\text { concentrazione } \\
\text { II tempo medio di residenza nel } \\
\text { plasma e tessuti è più lungo rispetto } \\
\text { alla endovenosa a bolo } \\
\text { Può essere effettuata facilmente a } \\
\text { domicilio da infermieri o da familiari } \\
\text { qualificati } \\
\text { É meno costosa della via } \\
\text { endovenosa per minori costi di } \\
\text { materiali e lavoro }\end{array}$ & 口 & $\begin{array}{l}\text { Possibile dolore, flogosi locale e } \\
\text { talora necrosi del tessuto con } \\
\text { formazione di ascessi } \\
\text { Non utilizzabile per posologie troppo } \\
\text { elevate e/o frequenti } \\
\text { Non consigliabile per trattamenti } \\
\text { prolungati } \\
\text { Non tutti gli antibiotici sono disponibili } \\
\text { in formulazione i.m. } \\
\text { Maggiori costi rispetto alla via orale } \\
\text { (vedi sopra) }\end{array}$ \\
\hline
\end{tabular}

Tabella VII

Vantaggi e svantaggi relativi della via orale, endovenosa e intramuscolare di somministrazione degli antibiotici per uso sistemico 
centuali di resistenze, le caratteristiche farmacocinetiche e farmacodinamiche e, infine, i vantaggi e gli svantaggi relativi della via orale, endovenosa e intramuscolare, come sintetizzati nella tabella VII.

Obiettivo di questa tappa decisionale è razionalizzare l'impiego degli antibiotici, soprattutto quando la scelta non può essere mirata ma solo empirica-ragionata, ed evitare che la scelta sia influenzata prevalentemente da fattori irrazionali. Come già sopra richiamato, nell'utilizzo degli antibiotici la razionalità non risiede tutta nella via orale piuttosto che nella via parenterale. Ovviamente, per molti pazienti l'uso di un antibiotico orale è la scelta razionale da attuare e l'utilizzo di un trattamento parenterale in questi casi può essere considerato eccessivo e non conveniente. Preme qui sottolineare ancora una volta come la scelta di un antibiotico iniettabile possa rappresentare un elemento importante che consente al medico di evitare il ricovero o di attuare un ricovero breve e continuare la cura a domicilio.

Dopo due o tre giorni di terapia antibiotica iniziale si pone il problema di una verifica dell' efficacia della cura: se le condizioni cliniche non sono migliorate o sono peggiorate, il paziente viene in genere ricoverato; se, invece, le condizioni cliniche sono stabilizzate o migliorate si può prendere in considerazione l'ipotesi di attuare una terapia sequenziale e di abbandonare la via parenterale per passare alla via orale. Tuttavia, la terapia sequenziale non sempre è praticabile: non sempre è disponibile una formulazione orale dell'antibiotico iniettabile; talvolta non esistono antibiotici orali con analogo spettro d'azione; per alcune infezioni e alcune localizzazioni è preferibile continuare tutto il ciclo terapeutico con lo stesso antibiotico per via parenterale; il paziente non può essere trattato per via orale.

Anche per i pazienti ricoverati si pone il problema di continuare con la terapia parenterale o di attuare una strategia sequenziale, una volta che le loro condizioni si sono stabilizzate. In ogni caso il raggiungimento e il consolidamento di una condizione clinica stabile tendente a migliorare rappresenta un elemento che consente di prendere in considerazione la dimissione del paziente: il passaggio ad un trattamento orale favorisce la dimissione precoce, ma anche la disponibilità di programmi di ADI o di ospedalizzazione domiciliare o la disponibilità del medico di Medicina Generale a continuare a domicilio la terapia parenterale può consentire la dimissione precoce di molti pazienti ed evitare che rimangano a lungo ricoverati solo per fare un trattamento parenterale.
Ultima tappa di un percorso decisionale tipico è determinare la fine della terapia antibiotica e quindi la durata complessiva del trattamento.

La Infectious Diseases Society of America (IDSA) alcuni anni or sono ha elaborato lineeguida per l'applicazione corretta e la gestione efficiente della terapia antibiotica iniettiva in ambito extra-ospedaliero (Community-based Parenteral Antibiotic Therapy o CoPAT) [38]. Recentemente queste linee-guida sono state aggiornate [39].

Queste linee-guida hanno lo scopo di fornire un supporto alle decisioni del medico sul quando, come e a quali pazienti praticare in modo ottimale la terapia antibiotica parenterale in ambito extra-ospedaliero. Le raccomandazioni sono, pertanto, indirizzate a tutti i medici, internisti, pediatri, medici di famiglia, e agli altri operatori interessati a praticare la terapia antibiotica iniettiva a domicilio o in altre strutture assistenziali extra-ospedaliere, come ambulatori medici, od organizzazioni appositamente progettate per questo tipo di intervento sanitario, denominate OPAT e diffuse negli Stati Uniti e in altri paesi.

In Italia la situazione è sensibilmente differente da quella degli Stati Uniti, ma la terapia antibiotica iniettiva viene applicata diffusamente in ambito extra-ospedaliero, prevalentemente a domicilio del paziente, sia in modo non strutturato su istanza estemporanea del medico curante di Medicina Generale, sia, sempre più frequentemente, in modo strutturato da equipe di sanitari organizzate dal SSN o dall'ospedale, secondo programmi di assistenza domiciliare integrata (ADI) o di ospedalizzazione domiciliare. Di seguito riprendiamo alcuni punti essenziali delle lineeguida IDSA, commentandoli alla luce della situazione italiana.

\section{Valutazione del paziente e criteri di selezione}

La decisione tecnica di attuare una terapia antibiotica iniettiva in ambito extra-ospedaliero spetta al medico curante, dopo essersi eventualmente consultato con altri medici specialisti, ad esempio un infettivologo, e dopo aver stabilito che la terapia antibiotica parenterale scelta è necessaria per trattare quella specifica infezione, che vi è una ragionevole prospettiva di poter controllare l'infezione con tale terapia e che vie alternative di somministrazione non sono indicate o non sono praticabili $[38,39]$.

Il principale obiettivo della terapia antibiotica iniettiva extra-ospedaliera è quello di consentire che i pazienti, che devono attuare questo tipo di trattamento, possano effettuarlo in piena sicurezza vivendo nel comfort della propria casa ed evitando gli inconvenienti, le com- 
plicazioni e i costi dell'ospedalizzazione prolungata. Questo obiettivo, tuttavia, non deve essere troppo enfatizzato al solo scopo di ridurre il consumo di risorse ospedaliere e dei relativi costi.

La selezione dei pazienti che possono essere gestiti in ambito extra-ospedaliero con terapia antibiotica iniettiva dovrebbe essere attuata dal medico curante in base ad alcuni semplici criteri generali, che le linee-guida IDSA riassumono nei punti indicati nella tabella VIII [38].

Secondo queste linee-guida, il primo criterio che il medico deve verificare nella selezione dei pazienti da avviare ad un trattamento antibiotico parenterale in ambito extra-ospedaliero è quello della necessità o meno del ricovero ospedaliero per motivi di sicurezza e salvaguardia della vita e dell'integrità del paziente e/o per la necessità di utilizzare tecniche diagnostiche, terapeutiche e assistenziali applicabili solo in ospedale.

In linea generale un paziente con un'infezione anche grave può essere trattato a domicilio o in altre strutture extra-ospedaliere solo quando le sue condizioni cliniche sono stabili o si sono stabilizzate e il rischio di peggioramenti improvvisi con minaccia per la vita può essere considerato minimo e accettabile. L'accertamento del rischio consiste nel determinare lo stato del processo infettivo, la sua fase evolutiva e tutte le condizioni cliniche concomitanti che possono incidere sulla sicurezza del paziente nell'ipotesi che la terapia antibiotica venga attuata o continuata in ambito extra-ospedaliero.

La terapia antibiotica iniettiva può essere iniziata a domicilio se il ricovero in ospedale non è necessario. Spesso la terapia antibiotica iniettiva viene iniziata in ospedale e continuata a domicilio o in altre strutture extra-ospedaliere dopo un semplice accesso al Pronto Soccorso $\mathrm{o}$ al Day-Hospital, o dopo un ricovero più o meno breve in corsia o addirittura in Terapia Intensiva. Anche pazienti terminali possono essere trattati a domicilio con terapia antibiotica iniettiva, se questo serve a migliorare la loro qualità di vita e ad aumentare il loro comfort.
La letteratura disponibile indica che molti tipi di infezione sono stati trattati con successo in ambito domiciliare o extra-ospedaliero mediante programmi di terapia antibiotica parenterale: infezioni delle basse vie respiratorie, endocarditi, osteomieliti, infezioni delle protesi articolari, infezioni della cute e dei tessuti molli, febbre del neutropenico, ascessi, e persino meningiti, ascessi cerebrali, ascessi epatici, oltre a molti altri tipi di infezioni [38,39].

In linea generale, pazienti con setticemia o infezioni focali quali meningite, endocardite, artrite settica o polmonite grave, dovrebbero essere ricoverati e trattati inizialmente in ospedale perché necessitano di cure mediche intensive e la loro condizione clinica può peggiorare rapidamente.

Quando le condizioni cliniche del paziente si stabilizzano e il decorso dell'infezione sembra avviarsi positivamente, il medico può valutare se esistono le premesse per una dimissione precoce e per poter continuare la terapia antibiotica iniettiva a domicilio. Questa ipotesi è particolarmente interessante per quelle infezioni, come le endocarditi e le osteomieliti, che richiedono cicli lunghi di terapia con antibiotici per via parenterale, effettuate un tempo quasi sempre solo in regime di ricovero ordinario. Il medico, tuttavia, deve conoscere molto bene quale sono i rischi di complicazioni anche per un paziente stabilizzato, rischi che variano in funzione della sede e dell'agente eziologico dell'infezione. Ad esempio, pazienti con endocardite valvolare sinistra da $S$. aureus possono sviluppare improvvisamente complicazioni anche quando le condizioni cliniche sono stabilizzate, mentre pazienti con endocardite da streptococchi possono essere gestiti a domicilio con minori rischi $[40,41]$.

Oltre alle condizioni cliniche del paziente, il medico deve verificare se vi sono le condizioni logistiche e organizzative per effettuare in sicurezza una terapia antibiotica iniettiva in ambito extra-ospedaliero. A domicilio la somministrazione di farmaci per via parenterale, soprattutto quando si deve usare la via endovenosa per infusione, richiede un minimo di

1. II tipo di infezione e soprattutto le condizioni cliniche del paziente non sono tali da richiedere il ricovero in ospedale e non necessitano di risorse assistenziali superiori a quelle disponibili a domicilio o nella struttura extra-ospedaliera nella quale viene attuata la terapia antibiotica iniettiva

2. II paziente o chi l'assiste è in grado di

a. attuare la terapia antibiotica iniettiva in modo sicuro ed efficace

b. seguire fedelmente le indicazioni e le raccomandazioni che gli vengono fornite

c. decidere di attuare la terapia iniettiva che gli viene proposta, dopo essere stato informato e aver discusso dei benefici, dei rischi e delle implicazioni economiche e aver dato un valido consenso, quando sia appropriato

3. Vi sono sistemi di comunicazione tra il paziente, chi l'assiste, il medico e altro personale medico tali da garantire un adeguato monitoraggio della terapia

4. L'ambiente domestico o della struttura è sicuro e adatto per effettuare la terapia antibiotica iniettiva

Tabella VIII Criteri generali per selezionare i pazienti che possono essere trattati con antibiotici iniettivi in ambito extra-ospedaliero (modificata da: Williams et al, 1997) [38] 
organizzazione assistenziale, medica e infermieristica. Ottimali sotto questo aspetto sono i programmi di Assistenza Domiciliare Integrata o di Ospedalizzazione Domiciliare messi in atto da alcune ASL o ASO. Con queste struttura operative è possibile effettuare trattamenti parenterali più complessi, utilizzare eventualmente sistemi di infusione programmata (pompe), gestire la sorveglianza di parametri clinici e laboratoristici necessari in relazione al tipo di infezione e al tipo di antibiotico. Se l'antibiotico è somministrabile per via intramuscolare l'impegno organizzativo è minore e spesso tra i familiari o i vicini si trovano persone qualificate per praticare un'iniezione intramuscolare.

Il livello di capacità collaborativa del singolo paziente deve essere attentamente valutato prima di iniziare un programma di terapia antibiotica iniettiva domiciliare.

La presenza di familiari capaci e disposti a dedicare al paziente un livello adeguato di assistenza infermieristica e igienica è condizione spesso sufficiente per poter avviare un trattamento iniettivo domiciliare con antibiotici sicuri e maneggevoli. La presenza di familiari o di assistenza privata è una condizione essenziale nel caso di pazienti in età pediatrica o di pazienti disabili.

In presenza di esigenze tecniche più complesse è necessario prevedere l'intervento di personale infermieristico appositamente addestrato per l'assistenza a domicilio. La partecipazione di personale infermieristico a programmi di terapia antibiotica iniettiva domiciliare è addirittura irrinunciabile quando il paziente è disabile e quando vi siano esigenze specifiche da soddisfare, come il controllo e la cura di accessi venosi centrali. Il numero di visite domiciliari degli infermieri può variare da un massimo di 1-2 al giorno ad un minimo di 1 ogni 2-3 o più giorni.

Oltre alla capacità collaborativa del paziente è opportuno che il medico valuti anche la sua affidabilità complessiva, l'assenza di abuso di sostanze, soprattutto per via endovenosa, e l'assenza di disturbi psichici che, oltre a ridurre la compliance, potrebbero costituire fattori di rischio di comportamenti pericolosi. Analogamente, la presenza nel nucleo familiare-abitativo di persone tossicomani o con disturbi psichiatrici gravi può rappresentare una controindicazione per l'effettuazione di un trattamento antibiotico iniettivo a domicilio.

L'effettuazione in sicurezza di trattamenti parenterali a domicilio del paziente richiede, infine, che vi sia la possibilità di comunicare, via telefono possibilmente, tra il paziente o suoi familiari e il medico o l'equipe medica che ha la responsabilità della terapia. Inoltre, l'ubicazione dell'abitazione del paziente do- vrebbe poter consentire un facile accesso ad ambulanze nel caso si rendesse urgente la necessità di un ricovero.

Infine, le condizioni igieniche dell' abitazione devono avere standard adeguati per ridurre al minimo il rischio di contaminazioni del materiale iniettato e delle vie di accesso venoso. Inoltre può essere indispensabile la presenza di un frigorifero dove conservare l'antibiotico.

Tutte queste condizioni ambientali e logistiche sono facilmente disponibili oggi in Italia, soprattutto nelle città. Qualche problema, tuttavia, può ancora presentarsi per pazienti che abitano in piccoli comuni montani, talvolta difficili da raggiungere soprattutto in periodi invernali, o in piccole isole del mediterraneo. Nelle aree urbane, invece, vi è un numero crescente di emarginati che vivono in case fatiscenti e in condizioni igieniche molto scadenti che non consentono di programmare una terapia antibiotica parenterale a domicilio.

\section{Scelta e gestione dell'antibiotico}

La scelta e la gestione dell'antibiotico parenterale a domicilio richiedono alcune considerazioni aggiuntive.

Quando si imposta una posologia è importante considerare non solo la farmacocinetica ma anche la farmacodinamica (o dinamica di killing) dell' antibiotico al fine di ottimizzare la probabilità di successo e ridurre il rischio di resistenze e di reazioni avverse.

Studi recenti hanno portato alla classificazione degli antibiotici in due gruppi in relazione alla loro farmacodinamica prevalentemente tempo-dipendente o concentrazione-dipendente (tabella IX) [42].

Utilizzando antibiotici che inducono una batteriocidia concentrazione-dipendente l'obiettivo della posologia dovrebbe essere quello di raggiungere concentrazioni il più possibile elevate, compatibilmente con problemi di tollerabilità e tossicità. Infatti è stato dimostrato che si ottiene un'efficacia clinica ottimale e si riduce il rischio di indurre resistenze quando il rapporto tra concentrazione plasmatica di picco e MIC del patogeno (Cmax/MIC) ha un valore superiore ad almeno 8-10 o quando il rapporto Area sotto la Curva delle Concentrazioni plasmatiche nelle 24 ore e MIC del patogeno (AUC24h/MIC) è di almeno 125.

Al contrario, quando si utilizzano antibiotici ad attività tempo-dipendente l'obiettivo della posologia dovrebbe essere quello di mantenere le concentrazioni sopra il valore della $\mathrm{MIC}$ il più a lungo possibile e comunque per non meno del 40-60\% dell' intervallo tra le dosi.

In linea generale, per ottimizzare il risultato terapeutico occorre impostare lo schema posologico e la modalità di somministrazione 


\begin{tabular}{|c|c|c|c|}
\hline Antibiotici & $\begin{array}{c}\text { Caratteristiche } \\
\text { farmacodinamiche }\end{array}$ & $\begin{array}{l}\text { Obiettivo della } \\
\text { posologia }\end{array}$ & $\begin{array}{l}\text { Parametri surrogati } \\
\text { dell'efficacia clinica }\end{array}$ \\
\hline $\begin{array}{l}\text { Aminoglicosidi } \\
\text { Fluorochinoloni } \\
\text { Metronidazolo }\end{array}$ & $\begin{array}{l}\text { Killing concentrazione-dipendente, } \\
\text { Post-Antibiotic Effect prolungato }\end{array}$ & $\begin{array}{l}\text { Ottenere } \\
\text { concentrazioni } \\
\text { elevate }\end{array}$ & $\begin{array}{l}\mathrm{Cmax} / \mathrm{MIC}>8-10 \\
\text { AUC24h/MIC }>125\end{array}$ \\
\hline $\begin{array}{l}\text { Penicilline } \\
\text { Cefalosporine } \\
\text { Aztreonam }\end{array}$ & $\begin{array}{l}\text { Killing tempo-dipendente, } \\
\text { Post-Antibiotic Effect nullo o } \\
\text { trascurabile }\end{array}$ & $\begin{array}{l}\text { Ottenere } \\
\text { concentrazioni >MIC } \\
\text { prolungate nel tempo }\end{array}$ & $\begin{array}{c}\text { Tempo supra-MIC }>40-60 \% \\
\text { intervallo dosi }\end{array}$ \\
\hline $\begin{array}{l}\text { Carbapenemi } \\
\text { Glicopeptidi } \\
\text { Clindamicina } \\
\text { Macrolidi }\end{array}$ & $\begin{array}{l}\text { Killing tempo-dipendente, } \\
\text { Post-Antibiotic Effect prolungato }\end{array}$ & $\begin{array}{c}\text { Ottenere } \\
\text { concentrazioni }>\mathrm{MIC} \\
\text { prolungate nel tempo }\end{array}$ & $\begin{array}{c}\text { Tempo supra-MIC }>40-60 \% \\
\text { intervallo dosi }\end{array}$ \\
\hline
\end{tabular}

dell'antibiotico scelto, seguendo in modo scrupoloso le indicazioni, le raccomandazioni e le avvertenze contenute nella scheda tecnica di registrazione.

In base alle attuali conoscenze sulla farmacodinamica degli antibiotici, gli aminoglicosidi dovrebbero essere somministrati in un'unica dose giornaliera mediante infusione endovenosa di breve durata in modo da ottenere un picco massimo di concentrazione elevata e sfruttare al meglio la loro attività battericida. Questo tipo di posologia, infatti, mentre aumenta l'efficacia terapeutica perché incrementa la probabilità di uccidere anche patogeni poco sensibili, non aumenta il rischio di ototossicità e di nefrotossicità, rischio che dipende dalla quantità totale di farmaco somministrato piuttosto che dalle concentrazioni plasmatiche raggiunte [43-45].

Questa strategia posologica, tuttavia, non è stata ancora documentata in modo esaustivo in tutti i tipi di infezione e in tutti i tipi di pazienti (ad esempio bambini, anziani, pazienti critici, donne in gravidanza, neutropenici, ustionati, insufficienti renali ed epatici, ecc.) [45]. D'altra parte la somministrazione di aminoglicosidi richiede comunque un' attenta valutazione della funzionalità renale del paziente, l'individualizzazione della posologia e una strategia complessiva di monitoraggio dei livelli plasmatici, nonché della funzionalità acustica ed eventualmente vestibolare, in caso di cicli terapeutici prolungati oltre i 10 giorni.

Lo schema posologico ottimale per somministrare antibiotici parenterali ad attività tempo-dipendente, come le beta-lattamine, dovrebbe consistere in una dose iniziale di carico a bolo o ad infusione rapida, seguita da un'infusione lenta a velocità costante: con la dose iniziale si inducono immediatamente concentrazioni plasmatiche superiori alla MIC dei patogeni, mentre con l'infusione lenta si mantengono le concentrazioni raggiunte, rimpiazzando la quota di farmaco eliminata in ogni frazione di tempo [46].
Può essere un grave errore iniziare ad infondere l'antibiotico senza una dose iniziale di carico: se la velocità di infusione è troppo bassa, le concentrazioni dell' antibiotico possono rimanere per troppo tempo inferiori alla MIC, risultare quindi inefficaci e determinare una selezione di ceppi resistenti.

La gestione di alcuni antibiotici comporta l'esecuzione di alcuni controlli di laboratorio e/o strumentali per verificare il livello di accumulo e l'eventuale presenza di reazioni avverse. Particolare cura deve prestare il medico nell'utilizzo degli aminoglicosidi per il rischio di nefrotossicità e soprattutto di ototossicità.

\section{CRITERI FARMACOECONOMICI}

Nella prospettiva, assai riduttiva, di contenimento della spesa farmaceutica, l'uso a domicilio di antibiotici iniettabili rappresenta un costo eccessivo da tagliare. In una prospettiva più ampia, tuttavia, la TAID presenta diversi potenziali vantaggi e solo pochi svantaggi per il paziente, per il SSN e per la società. Pertanto, una valutazione più ponderata del valore della TAID dovrebbe basarsi sull' analisi dei costi incrementali indotti e sul confronto di questi costi incrementali con il guadagno in benefici sanitari, prendendo come riferimento l'alternativa del ricovero ospedaliero.

Non è obiettivo di questo lavoro affrontare l'analisi dettagliata e ragionieristica dei costi differenziali correlati alla TAID al fine di giungere ad una precisa stima monetaria. Interessa, invece, tentare un' analisi dei valori da attribuire a tale scelta considerando tre diverse fondamentali prospettive, quella del paziente, del SSN e della società.

L'analisi dei valori è una tecnica di analisi decisionale che consiste nell' assegnare un insieme di valori ad una determinata scelta in ambito sanitario, i cui outcomes, ossia costi e benefici, possono variare a seconda della prospettiva adottata. Il presupposto teorico su
Tabella IX Correlazione tra fattori farmacodinamici ed efficacia clinica degli antibiotici (modificata da: Craig WA, 1995) [42] 
cui tale tecnica si basa consiste nella constatazione che un risultato che appare positivo ad un decisore, ad esempio al SSN, possa rivestire un significato differente per gli altri attori coinvolti nella decisione, ad esempio il paziente o la società nel suo complesso $[47,48]$. Scopo di questo tipo di analisi è quello di fornire un quadro completo delle ricadute di una scelta nelle diverse prospettive adottate: a tal fine è utile costruire una matrice a tre dimensioni in cui, per ogni decisore considerato, si considerano gli incroci tra tipo di risultato, clinico e economico, ed il valore positivo e negativo di tali risultati.

Nella tabella X è riportata la matrice costruita per analizzare i valori clinico ed economico, positivi e negativi, della TAID nella prospettiva del paziente, del SSN e della società italiana. I giudizi espressi derivano dall' esame della letteratura disponibile, dal contenuto delle citate linee-guida IDSA e dalle considerazioni sopra esposte.

Una terapia antibiotica iniettiva domiciliare, condotta secondo adeguati canoni tecnicoscientifici ed organizzativi, non comporta un deterioramento dei risultati sanitari complessivi [38]. Al contrario, secondo alcuni autori la TAID può comportare qualche vantaggio sanitario significativo, come la riduzione del rischio di infezioni ospedaliere, un minor numero di infezioni e un minor rischio di mortalità e di complicazioni gravi associate all'uso di cateteri [49]. Questo risultato, tuttavia, dovrebbe essere meglio documentato e confermato da ulteriori evidenze.

Per il paziente la TAID rappresenta soprattutto un'opportunità per evitare il disagio dell'ospedale ed effettuare comodamente la terapia antibiotica iniettiva a casa, circondato dall'affetto e dal supporto dei familiari. Ovviamente questo risultato non è valido per tutti i pazienti, ad esempio non può essere necessariamente valido per i pazienti che vivono da soli o che hanno situazioni familiari non armoniche; pertanto alcuni pazienti possono preferire un ricovero ospedaliero piuttosto che dover affrontare le pur minime complicazioni che un trattamento iniettivo domiciliare comporta.

In definitiva, quindi, ad un'analisi conservativa attuata nella prospettiva del SSN, del paziente e della società, i benefici sanitari della TAID possono essere considerati solo debolmente superiori a quelli ottenibili con il ricovero. Nell'ipotesi di voler condurre un' analisi costo/efficacia dovremmo senz'altro assumere che la TAID abbia un valore sanitario complessivo equivalente a quello dell'analogo trattamento parenterale effettuato in regime di ricovero ospedaliero. Su tale premessa sarebbe pertinente utilizzare la tecnica "minimizzazione dei costi" per decidere quale delle due alternative sia più conveniente nella prospettiva del SSN o della società.

\begin{tabular}{|c|c|c|c|c|}
\hline & \multicolumn{2}{|c|}{ Valore Clinico } & \multicolumn{2}{|c|}{ Valore Economico } \\
\hline & Positivo & Negativo & Positivo & Negativo \\
\hline $\begin{array}{l}\text { Per il } \\
\text { paziente }\end{array}$ & $\begin{array}{l}\text { Effettuare la terapia a } \\
\text { casa propria con maggiore } \\
\text { comfort ed il supporto dei } \\
\text { familiari }\end{array}$ & $\begin{array}{l}\text { Rottura della routine } \\
\text { familiare } \\
\text { Aumento dello stress }\end{array}$ & $\begin{array}{l}\text { ( Possibilità di ritornare al lavoro } \\
\text { o a scuola più precocemente } \\
\text { (riduzione dei costi indiretti a } \\
\text { carico del paziente) }\end{array}$ & $\begin{array}{l}\text { ( Possibile aumento delle } \\
\text { compartecipazioni ai costi } \\
\text { diretti sanitari (ticket, } \\
\text { farmaci, ecc.) } \\
\text { u Possibile aumento delle } \\
\text { piccole spese (costi diretti } \\
\text { non sanitari a carico del } \\
\text { paziente) }\end{array}$ \\
\hline $\begin{array}{l}\text { Per il } \\
\text { SSN }\end{array}$ & $\begin{array}{l}\text { u Uguale o migliore } \\
\text { efficacia globale } \\
\text { u Diminuzione del rischio } \\
\text { di infezioni ospedaliere } \\
\text { u Minor numero di } \\
\text { infezioni da cannula e } \\
\text { flebiti e minor mortalità } \\
\text { associata a tali } \\
\text { complicazioni }\end{array}$ & $\begin{array}{l}\text { Q Maggior rischio di } \\
\text { scarsa compliance alla } \\
\text { terapia rispetto al ricovero } \\
\text { a Rischio di interruzione } \\
\text { della terapia endovenosa } \\
\text { u Uso scorretto o } \\
\text { improprio dell'accesso } \\
\text { venoso }\end{array}$ & $\begin{array}{l}\text { u Minori costi diretti totali } \\
\text { u Minori costi per ricoveri } \\
\text { ospedalieri: } \\
\text { - minor numero di ricoveri } \\
\text { - minor durata della degenza } \\
\text { - maggior turnover dei posti letto } \\
\text { - utilizzo più efficiente dei posti } \\
\text { letto }\end{array}$ & $\begin{array}{l}\text { ( Maggior costo di } \\
\text { acquisto dei farmaci (salvo } \\
\text { dispensazione } \\
\text { ospedaliera) } \\
\text { u Maggior costo } \\
\text { dell'assistenza domiciliare } \\
\text { (infermieri, ecc.) }\end{array}$ \\
\hline $\begin{array}{l}\text { Per la } \\
\text { società }\end{array}$ & $\begin{array}{l}\text { u Uguale o migliore } \\
\text { risultato sanitario } \\
\text { complessivo }\end{array}$ & ( Nessuno apparente & $\begin{array}{l}\text { ( Maggiore efficienza } \\
\text { complessiva dei costi correlati alle } \\
\text { infezioni batteriche } \\
\text { u Maggiore offerta di servizi } \\
\text { sanitari e assistenziali } \\
\text { u Riduzione dei costi indiretti da } \\
\text { perdita di produttività }\end{array}$ & $\begin{array}{l}\text { Necessità di formazione } \\
\text { specifica per gli operatori, i } \\
\text { familiari e anche per i } \\
\text { pazienti }\end{array}$ \\
\hline
\end{tabular}

Tabella $\mathrm{X}$

Matrice dei valori clinico ed economico proposta per analizzare il tema della terapia antibiotica iniettiva domiciliare (TAID) nella prospettiva del paziente, del SSN e della società italiana 
Nella prospettiva del SSN, il valore economico della TAID appare globalmente positivo. Infatti, a fronte di un maggior costo d'acquisto dei farmaci e ad un maggior costo dell'assistenza domiciliare, la TAID consente di ridurre i costi diretti sanitari a carico del SSN, soprattutto diminuendo il numero di ricoveri e la durata delle degenze. Questo risultato, tuttavia, per poter essere pienamente apprezzato dal SSN, ossia dalle ASL, deve poter indurre una razionalizzazione del sistema ospedaliero attraverso un ridimensionamento dei posti letto per acuti e una diversa qualificazione dei posti letto eventualmente in esubero, ricollocandoli in settori attualmente carenti o sottodimensionati, come la riabilitazione e la lungodegenza. Nell' attesa che questo avvenga, un eventuale aumento delle terapie antibiotiche parenterali domiciliari rischia di essere percepito dal SSN come puro incremento dei costi, e in particolare come puro incremento della spesa farmaceutica.

I costi diretti sanitari della TAID a carico del SSN sono inferiori a quelli del ricovero anche per la riduzione dei casi di infezione ospedaliera, il minor numero di infezioni del catetere e, in minima parte, per una maggior compartecipazione alla spesa farmaceutica e diagnostica, attualmente richiesta al paziente ambulatoriale.

In effetti, nell'attuare un programma di TAID, il paziente deve sopportare un sensibile aggravio di spesa, dovuto in parte al sistema di compartecipazione (es. ticket) su alcuni costi diretti sanitari ed in parte ai costi diretti non sanitari (piccole spese indotte da questo tipo di terapia) che pesano totalmente su di lui e sui suoi familiari. A fronte di questi aggravi economici, il paziente e la sua famiglia possono beneficiare di una certa riduzione dei costi indiretti, resa possibile dalla TAID. Infatti, in molti casi la TAID consente di riprendere precocemente una qualche attività lavorativa, di tornare a scuola ed evitare l'utilizzo di baby-sitter o di altro personale ausiliario.

I benefici economici dei pazienti e del SSN si riverberano complessivamente sulla società. La TAID può comportare un sensibile risparmio sui costi indiretti totali che gravano sulla società. Inoltre può comportare altri benefici sociali indiretti, difficilmente monetizzabili, consistenti in una maggiore varietà di offerta di servizi sanitari e assistenziali e in una maggiore efficienza complessiva dell'allocazione delle risorse sanitarie. Infine, organizzazioni di TAID come programmi di ADI mobilitano gruppi di volontariato e contribuiscono a creare un clima sociale collaborativo, aperto e meno egoistico.

In conclusione, 1 'analisi dei valori evidenzia come la TAID possa essere maggiormente co- sto/efficace rispetto all' analogo trattamento in regime ospedaliero. Questo risultato dipende prevalentemente dal risparmio conseguibile con la riduzione dei ricoveri e la dimissione precoce dei pazienti dall'ospedale [50-52].

\section{Entità dei risparmi possibili con la TAID}

Non è facile stabilire con precisione quanto costi di meno la TAID rispetto al trattamento in regime di ricovero. Innanzitutto non è facile stabilire quanto costi mediamente una giornata di degenza in ospedale per un paziente affetto da una grave infezione che necessita del ricovero. Si consideri, inoltre, che possono esistere notevoli differenze di costo medio delle degenze in relazione ai diversi tipi di infezione.

I costi della TAID possono variare notevolmente in ragione del tipo di organizzazione coinvolta, del numero di medici e infermieri impiegati, del numero di accessi al domicilio del paziente necessari, delle tecniche e dei dispositivi impiegati per la somministrazione parenterale degli antibiotici (es. CVC, pompe per infusione, ecc.).

Diverse valutazioni sono state pubblicate su questo tema relativamente all'assetto assistenziale degli Stati Uniti, come riporta la tabella XI [53-56]. Una rassegna di questi lavori ha dimostrato come negli Stati Uniti i vari programmi di OPAT, condotti in anni differenti e in luoghi differenti, comportino un risparmio percentuale, rispetto al ricovero ospedaliero, sorprendentemente uniforme, variabile dal $63 \%$ al $70 \%$. Infatti il costo giornaliero medio per pazienti in trattamento OPAT è circa un terzo del costo medio giornaliero dello stesso trattamento in ospedale [57].

Questi risultati non possono essere trasferiti direttamente alla situazione italiana, data la diversa struttura del SSN e la molteplicità e multiformità delle esperienze di TAID finora registrate in Italia: si va dalla semplice somministrazione intramuscolare autogestita, minimamente costosa, a programmi di ADI o di ospedalizzazione domiciliare, notevolmente più costosi.

Una stima attuata alcuni anni or sono sul trattamento delle riacutizzazioni della bronchite cronica ha portato gli autori a concludere che con il costo di un ricovero ospedaliero (valore del relativo DRG) si potrebbero trattare a domicilio per via parenterale da un minimo di 6 ad un massimo di 25 pazienti [58]. Si tratta di stime, verosimilmente molto ottimistiche, valide per la somministrazione intramuscolare autogestita. I programmi di ADI e di ospedalizzazione domiciliare probabilmente comportano risparmi meno clamorosi ma tuttavia molto interessanti, dell' ordine di grandezza simile a quelli registrati negli Stati Uniti. 


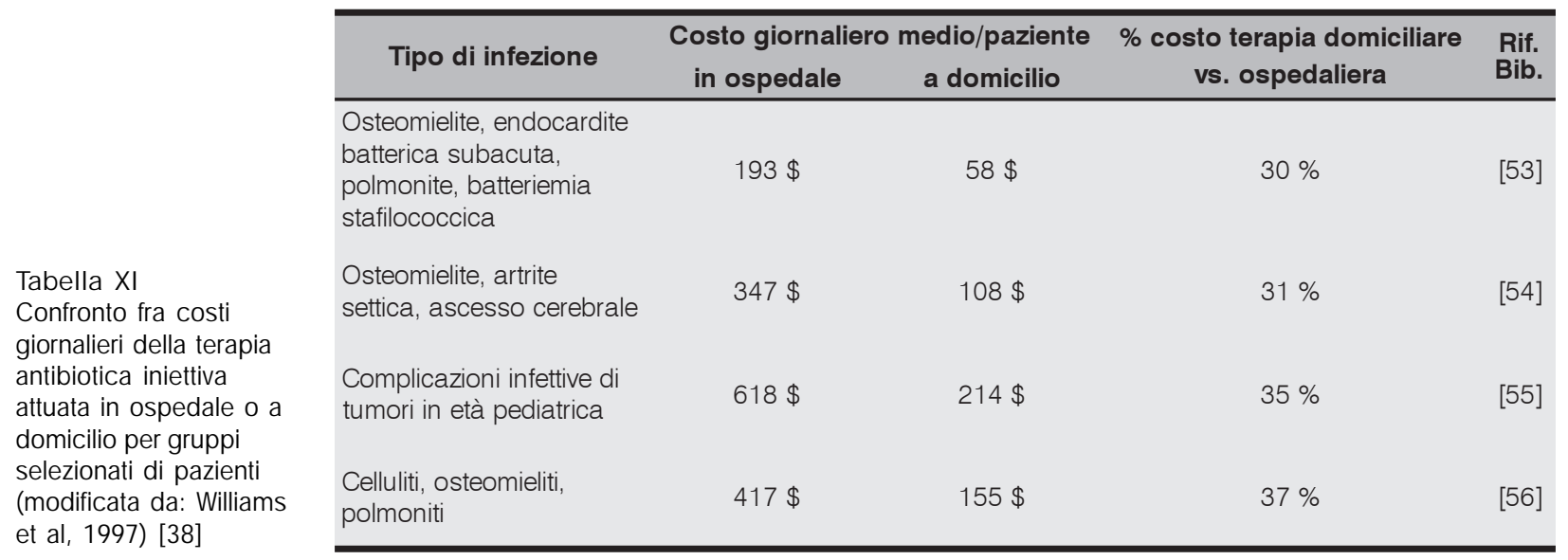

La scelta razionale dell' antibiotico è uno dei principali fattori critici capaci di determinare se il risultato farmacoeconomico sarà più o meno favorevole per il SSN e la società. Questo assunto è ancora più valido nel caso di terapie antibiotiche parenterali attuate fin dall'inizio a domicilio o continuate a domicilio del paziente dopo un iniziale periodo di ospedalizzazione.

Il costo di acquisto dell'antibiotico parenterale rappresenta solo una modesta quota dei costi totali di gestione delle infezioni batteriche moderate o gravi. Quando la terapia è impostata in modo razionale, il costo di acquisto più elevato dei farmaci iniettabili viene facilmente controbilanciato da tassi di guarigione più elevati, da un minor uso di antimicrobici aggiuntivi, da una riduzione dei ricoveri attuati in prima istanza o come complicanze, da una riduzione di giorni di degenza in ospedale e, talvolta, da una durata complessiva inferiore della malattia e dell' assenza dal lavoro.

L'attuale tendenza ad usare, anche sul territorio, antibiotici iniettabili ad ampio spettro, più costosi, può essere giustificata dalla necessità di vincere la resistenza batterica di patogeni difficili, ormai presenti anche in ambito extra-ospedaliero. Tuttavia, nell'ambito dei numerosi antibiotici ad ampio spettro oggi disponibili, occorre fare una qualche differenza fra quelli che possono favorire e quelli che possono limitare l'insorgenza di resistenze, fra quelli che sono maneggevoli e quelli che sono più difficili da usare.

A tal proposito, piperacillina/tazobactam riveste una posizione particolare per il suo ampio spettro, la minore tendenza ad indurre ceppi resistenti e la buona tollerabilità.

Diversi studi hanno indicato che una riduzione dell'uso generalizzato di cefalosporine a favore delle beta-lattamine protette può portare ad un recupero della sensibilità batterica [59-62].
L'impiego di piperacillina/tazobactam come farmaco di prima linea al posto delle cefalosporine di $\mathrm{III}^{\circ}$ generazione può limitare l'insorgenza di resistenze batteriche ESBL e GRE, conservando intatta l' attività di farmaci preziosi come la vancomicina e la teicoplanina.

L'insieme di questi risultati e soprattutto la riduzione della resistenza batterica comporta anche un risparmio di risorse a favore del SSN e della società [59].

Una recente rassegna farmacoeconomica ha sottolineato che l'uso di piperacillina/ tazobactam potenzialmente riduce i costi globali di trattamento delle infezioni batteriche moderate e gravi, alzando il tasso di successo iniziale e riducendo di conseguenza la durata delle degenze ospedaliere e l'uso di antibiotici aggiuntivi. Gli studi farmacoeconomici pubblicati indicano che l'uso di piperacillina/tazobactam è maggiormente costo efficace, rispetto ai regimi antibatterici standard, nel trattamento delle infezioni delle basse vie respiratorie, delle infezioni addominali, nelle infezioni della cute e tessuti molli e negli episodi febbrili del paziente neutropenico [63].

\section{CONCLUSIONI}

La terapia antibiotica iniettiva a domicilio è una opzione che il medico curante deve poter utilizzare, secondo linee-guida razionali e condivise, nell'interesse prioritario del paziente e a beneficio del SSN e della società.

Le nuove note AIFA 55 e 56 da un lato pongono un freno all'abuso e orientano il medico ad un uso più razionale degli antibiotici iniettabili sul territorio, dall'altro consentono di gestire a domicilio anche pazienti con infezioni batteriche non banali e favoriscono l'implementazione di programmi di TAID più strutturati, come l'ADI e l'ospedalizzazione domiciliare, contribuendo 
a creare le condizioni per un significativo risparmio della spesa sanitaria pubblica.

L'opportunità di utilizzare la TAID, oltre che da opzioni personali del medico e del paziente, deriva dalle evidenze fornite recentemente dall'epidemiologia microbiologica delle infezioni acquisite a domicilio e dalla valutazione dei significativi vantaggi economici per il SSN e per la società indotti dalla TAID nel caso in cui possa evitare il ricovero o consenta la riduzione delle giornate di degenza.

La scelta della TAID da parte del medico deve essere una decisione tecnicamente valida e ben ponderata, caso per caso. Le linee-guida disponibili nella letteratura internazionale sono un utile riferimento per ren- dere razionale le scelte del medico, evitare abusi e misusi, iper- ed ipo-utilizzazioni degli antibiotici parenterali, mediante un processo di personalizzazione del trattamento sulla base delle condizioni cliniche, psicologiche e sociali del paziente.

Quando il medico, seguendo linee-guida razionali, riesce onestamente ad attribuire ad ogni paziente la terapia antibiotica più adeguata ai suoi specifici bisogni di cura, persegue anche un obiettivo di equità e di efficienza allocativa delle risorse sanitarie. Questa soluzione può non corrispondere a quella proposta da strategie di contenimento della spesa farmaceutica, ma è sicuramente più rispettosa dei bisogni reali del singolo paziente.

\section{BIBLIOGRAFIA}

1. Le nuove Note AIFA. BIF n. 3-4, 2004, Ministero della Salute, AIFA. (http://www.ministerosalute.it/medicinali/ informazioneFarmaci/noteCUF.jsp)

2. OsMED, L'uso dei farmaci in Italia: Rapporto Nazionale 2003. Ministero della Salute, giugno 2004 (http:// www.ministerosalute.it/medicinali/osmed/osmed.jsp)

3. OsMED, L'uso dei farmaci in Italia: Rapporto Nazionale gennaio-luglio, 2004. AIFA, Ministero della Salute, Novembre 2004. (http://www.ministerosalute.it/medicinali/osmed/osmed.jsp)

4. Cars O, Molstad S, Melander A. Variation in antibiotic use in the European Union. Lancet 357:1851-1853, 2001.

5. Halls GA. The management of infections and antibiotic therapy: a European survey. J Antimicrob Chemother 31 : 985-1000, 1993.

6. De Lalla F, Eandi M. Indagine sull'uso di antibiotici nel trattamento delle infezioni delle base vie respiratorie negli ospedali italiani: considerazioni farmacoeconomiche. Giornale Italiano di Chemioterapia 45: 59-87, 1998

7. Eandi M. Farmacoeconomia della terapia antibiotica iniettiva extra-ospedaliera. In: "TAID, Terapia Antibiotica Iniettiva Domiciliare: -Clinica, Farmacologia, Farmacoeconomia", S. Esposito, M Eandi, A. Novelli, T Mazzei \& G Bettoncelli eds., Edimes , Pavia, 2001.

8. Schaberg T, Gialdroni-Grassi G, Huchon G, et al. An analysis of decisions by European general practitioners to admit to hospital patients with lower respiratory tract infections. The European Study Group of Community Acquired Pneumonia (ESOCAP) of the European Respiratory Society. Thorax 51:1017-22, 1996.

9. Chambers HF. The changing epidemiology of Staphylococcus aureus? Emerg Infect Dis 7: 178-182, 2001.

10. Berman D, Eisner W, Kreiswirth B. Community-acquired methicillin-resistant Staphylococcus aureus infection. N Eng J Med 329:329, 1896, 1993.

11. Pate K, Nolan R, Bannermann T, et al. Methicillin-resistant Staphylococcus aureus in the community. Lancet 346: $978,1995$.

12. Herold BC, Immergluck LC, Maranan MC, et al. Community-Acquired Methicillin-Resistant Staphylococcus aureus in children with no identified predisposing risk. JAMA 279: 593-598, 1998.

13. Gorak E, Yamada S, Brown J. Community-acquired methicillin-resistant Staphylococcus aureus in hospitalized adults and children without known risk factors. Clin Infect Dis. 29 : 797-800, 1999.

14. Kallen A, Driscoll T, Thornton S, et al. Increase in community-acquired methicillin-resistant at a Naval Medical Center. Infect Control Hosp Epidemiol 21: 223-226, 2000. 
15. Stevens DL. Community-Acquired Resistant Staphylococcus aureus infections: increasing virulence and emerging resistance in the new millennium. Curr Opin Infect Dis 16: 189-191, 2003.

16. Eady EA, Cove JH. Staphylococcal resistance revisited: Community-Acquired Methicillin-Resistant Staphylococcus aureus - an emerging problem for the management of skin and soft tissue infections. Curr Opin Infect Dis 16: 103$124,2003$.

17. Stefani S, Varaldo PE. Epidemiology of methicillin-resistant staphylococci in Europe. Clin Microbiol Infect 9: 1179$86,2003$.

18. Salgado CD, Farr BM, Calfee DP. Community-Acquired Methicillin-Resistant Staphylococcus aureus: a Metaanalysis of prevalence and risk factors. Clin Infect Dis 36: 131-139, 2003.

19. Diekema DJ, Pfallere MA, Schmitz FJ, et al. Survey of infections due to Staphylococcus species: frequency of occurrence and antimicrobila susceptibility of isolates collected in the Unietd States, Canada, Latin America, Europe, and the Western Pacific region for the SENTRY Antimicrobial Surveillance Program, 1997-1999. Clin Infect Dis 32 (suppl 2): 114-132, 2001.

20. Sanguinetti CM, De Benedetto F, Miragliotta G, DEDALO Study Group. Bacterial agents of lower respiratory tract infections (LRTIs), b-lactamase production, and resistance to antibiotics in elderly people. Int $\mathrm{J}$ Antimicrob Agents 16: 467-471, 2000.

21. Felmingham D, Grunenberg RN. The Alexander Project 1996-1997: latest susceptibility data from this international study of bacterial pathogens from community-acquired lower respiratory tract infections. J Antimcrob Chemother 45: 191-203, 2000.

22. PROTEKT Study Database. (http://www.proetkt.org)

23. Canton R, Loza E, Morosini MI, et al. Antimicrobial resistance amongst isolates of Streptococcus pyogenes and Staphylococcus aureus in the PROTEKT antimicrobial surveillance programme during 1999-2000. J Antimicrob Chemother 50 (suppl 1): 9-24, 2002.

24. Hoban D, Felmingham D. The PROTEKT surveillance study: antimicrobial susceptibility of Haemophilus influenzae and Moraxella catarrhalis from community-acquired respiratory tract infections. J Antimicrob Chemother 50 (suppl 1): 49-59, 2002.

25. Hoban DJ, Doern GV, Fluit AC, et al. Worldwide prevalence of antimicrobial resistance in Streptoicoccus penumoniae, Haemophilus influenzae, and Moraxella catarrhalis in the SENTRY Antimicrobial Surveillance Program $1997-$ 1999. Clin Infect Dis 32 (Suppl 2): S81-S93, 2001.

26. Felmingham D, Feldman C, Hryniewicz W, et al. Surveillance of resistance in bacteria causing community-acquired respiratory tract infections. Clin Microbiol Infect 8(Suppl2): 12-42, 2002.

27. Schito GC, Mannelli S, Marchese A, et al. Resistenza ai farmaci antimicrobici in Streptococcus pneumoniae circolanti nel 1999 in Italia. GIMMOC 4: 13-30, 2000.

28. Bronzwaer SL, Cars O, Buchholz U, et al. A European Study on the relationship between antimicrobial use and antimicrobial resistance. Emerg Infect Dis 8: 278-282, 2002.

29. Baquero F, Negri MC. Strategies to minimize the development of antibiotic resistance. J Chemother 9(Suppl 3): 2737, 1997.

30. Cornaglia G, Fontana R. Epidemiological survey of bacterial resistance in upper respiratory tract infections in Italy. Int J Antimicrob Agents 16: 259-262, 2000.

31. Cazzola M, Matera MG, Noschese P. Parenteral antibiotic therapy in the treatment of lower respiratory tract infections. Strategies to minimize the development of antibiotic resistance. Pulm Pharmacol Ther 13 : 249-256, 2000.

32. Borer A, Gilad J, Menashe G, et al. Extended-Spectrum Beta-Lactamase-Producing Enterobacteriaceae strains in community-acquired bacteriemia in Southern Israel. Med Sci Monit 8: 44-47, 2002.

33. Spanu T, Luzzaro F, Perilli M, et al. Occurence of Extended-Spectrum Beta-Lactamases in members of the family Enterobacteriaceae in Italy: implications for resistance to beta-lactams and other antimicrobial drugs. Antimicrob Agents Chemother 46: 196-202, 2002.

34. Stefani S, Pini B, Mezzatesta M, Perilli M, Rossolini GM, Amicosante G, Toniolo A, Luzzaro F. Italian Nationwide Survey: Extended Spectrum Beta-Lactamases in Isolates of Enterobacteriaceae from Inpatients and Outpatients. Abstract $44^{\circ}$ ICAAC, Washington DC 2004 
35. Schwaber MJ, Graham CS, Sands BE, et al. Treatment with a broad-spectrum cephalosporin versus piperacillintazobactam and the risk for isolation of broad-spectrum cephalosporin-resistant Enterobacter species. Antimicrob Agents Chemother 47: 1882-6, 2003.

36. Bantar C, Vesco E, Heft C, Salamone F, Krayeski M, Gomez H, Coassolo MA, Fiorillo A, Franco D, Arango C, Duret F, Oliva ME. Replacement of broad-spectrum cephalosporins by piperacillin-tazobactam: impact on sustained high rates of bacterial resistance. Antimicrob Agents Chemother. 2004 Feb;48(2):392-5

37. Serra R. Dati su file personali.

38. Williams DN, Rehm SJ, Tice AD, et al. Practice guidelines for community-based parenteral anti-infective therapy. Clin Infect Dis 25: 787-801, 1997.

39. Tice AD, Rehm SJ, Dalovisio JR, Bradley JS, Martinelli LP, Graham DR, Gainer RB, Kunkel MJ, Yancey RW, Williams DN; IDSA. Practice guidelines for outpatient parenteral antimicrobial therapy. IDSA guidelines. Clin Infect Dis. 2004 Jun 15;38(12):1651-72

40. Rehm SJ, Tomford JW, Longworth DL, et al. Home Intravenous Antibiotic Therapy (HIVAT) for endocarditis. Abstract n. 18 in: Proceedings 30 ${ }^{\text {th }}$ Annual Meeting of IDSA, 1992.

41. Stamboulian D, Bonvehi P, Arevalo C, et al. Antibiotic management of outpatients with endocarditis due to peneicillin-susceptible streptococci. Rev Infect Dis 13 (Suppl. 2): S160-S163, 1991.

42. Craig WA. Antibiotic selection factors and description of a hospital-based outpatient antibiotic therapy program in the USA. Eur J Clin Microbiol Infect Dis 14: 636-642, 1995.

43. Mattie H, Craig WA, Pechére JC. Determinants of efficacy and toxicity of aminoglycosides. J Antimicrob Chemother 24: 281-293, 1989.

44. Barza M, Ioannidis JPA, Cappeleri JC, et al. Single or multiple daily doses of aminoglycosides: a meta-analysis. BMJ 312: 338-345, 1996.

45. Dew RBIII, Sulsa GM. Once-daily aminoglycoside treatment. Infect Di Clin Pract 5: 12-24, 1996.

46. Craig WA, Ebert SC. Continuous infusion of beta-lactam antibiotics. Antimicrob Agents Chemother 36: 2577-2583, 1992.

47. Kahneman D, Twersky A. Choices, values, and frames. Am Psychologist 1984;39:341-450.

48. McGlynn EA et al. Quality-of-care research in mental health: responding to the challenge. Inquiry. 1988 Spring;25(1):157-70.

49. Graham DR, Keldermans MM, Klemm LW, et al. Infectious complications among patients receiving home intravenous therapy with peripheral, central, or peripherally placed central venous catheters. Am j Med 91 (Suppl. 3B): 95S100S, 1991.

50. Poretz DM. The infusion center: a model for outpatient parenteral antibiotic therapy. Rev Infect Dis 13 (Suppl. 2): S142-146, 1991.

51. Grizzard MB, Harris G, Karns H. Use of outpatient parenteral antibiotic therapy in a health maintenance organization. Rev Infect Dis 13 (Suppl. 2): S174-179, 1991.

52. Eisenmberg JM, Kitz DS. Savings from outpatient antibiotic therapy for osteomyelitis: economic analysis of a therapeutic strategy. JAMA 255: 1584-1588, 1986.

53. Stiver HG, Telford GO, Mossey JM, et al. Intravenous antibiotic therapy at home. Ann Intern Med 89: 690-693, 1978.

54. Chamberlain TM, Lehman ME, Groh MJ, et al. Cost analysis of a home intravenous antibiotic program. Am J Hosp Pharm 45: 2341-2345, 1988.

55. Wienikowski JT, Rothney M, Dawson S, et al. Evaluation of a home intravenous antibiotic program in pediatric oncology. Am J Pediatr Hematol Oncol 13: 144-147, 1991.

56. Williams DN, Bosch D, Boots J, et al. Safety, efficacy and cost savings in an outpatient intravenous antibiotic program. Clin Ther 15: 169-179, 1993.

57. Grainger-Rousseau TJ, Segal R. Economic, clinical and psycho-social outcomes of home infusion therapy: a review of published studies. Pharm Pract Manag Quart 15: 57-77, 1995.

58. Berto P. Comunicazione personale al Convegno "Le Infezioni batteriche degli anni '90 - Attualità terapeutiche" Napoli 23-25 gennaio 1997. 
59. Yates RR. New intervention strategies for reducing antibiotic resistance, Chest 115 (Suppl): 24S-27S, 1999.

60. Landman D, Chockalingam M, Quale JM. Reduction in the incidence of methicillin-resistant Staphylococcus aureus and ceftazidime-resistant Klebsiella pneumoniae following changes in a hospital antibiotic formulary. Clin Infect Dis 27: 81-83, 1999.

61. Rice LB. A silver bullet for colonization and infection with methicillin-resistant Staphylococcus aureus still eludes us. Clin Infect Dis 28: 1067-1070, 1999.

62. Smith DW. Decreased antimicrobial resistance after changes in antibiotic use. Pharmacotherapy 19 (Suppl): 129S132S, 1999.

63. Young M, Plosker GL. Piperacillin/tazobactam: rassegna del suo impiego nelle infezioni batteriche da moderate a gravi. Pharmacoeconomics 19: 1135-1175, 2001. 